\title{
The Lon protease temporally restricts polar cell differentiation events during the Caulobacter cell cycle
}

\author{
Deike J Omnus ${ }^{\dagger}$, Matthias J Fink ${ }^{\dagger}$, Klaudia Szwedo, Kristina Jonas*
}

Science for Life Laboratory and Department of Molecular Biosciences, The WennerGren Institute, Stockholm University, Stockholm, Sweden

\begin{abstract}
*For correspondence:
kristina.jonas@su.se

'These authors contributed equally to this work
\end{abstract}

Competing interest: The authors declare that no competing interests exist.

Funding: See page 20

Received: 14 September 2021

Accepted: 22 September 2021

Published: 25 October 2021

Reviewing Editor: Sonja V Albers, University of Freiburg, Germany

(c) Copyright Omnus et al. This article is distributed under the terms of the Creative Commons Attribution License, which permits unrestricted use and redistribution provided that the original author and source are credited.

\begin{abstract}
The highly conserved protease Lon has important regulatory and protein quality control functions in cells from the three domains of life. Despite many years of research on Lon, only a few specific protein substrates are known in most organisms. Here, we used a quantitative proteomics approach to identify novel substrates of Lon in the dimorphic bacterium Caulobacter crescentus. We focused our study on proteins involved in polar cell differentiation and investigated the developmental regulator StaR and the flagella hook length regulator FliK as specific Lon substrates in detail. We show that Lon recognizes these proteins at their C-termini, and that Lon-dependent degradation ensures their temporally restricted accumulation in the cell cycle phase when their function is needed. Disruption of this precise temporal regulation of StaR and FliK levels in a $\Delta l o n$ mutant contributes to defects in stalk biogenesis and motility, respectively, revealing a critical role of Lon in coordinating developmental processes with cell cycle progression. Our work underscores the importance of Lon in the regulation of complex temporally controlled processes by adjusting the concentrations of critical regulatory proteins. Furthermore, this study includes the first characterization of FliK in C. crescentus and uncovers a dual role of the $\mathrm{C}$-terminal amino acids of FliK in protein function and degradation.
\end{abstract}

\section{Introduction}

Intracellular proteolysis is a critical process in all cell types that is carried out by dedicated proteases. By removing damaged and non-functional proteins, proteases are necessary for maintaining protein homeostasis, in particular under stress conditions that threaten the proteome. Additionally, proteases have important regulatory roles in precisely adjusting the amounts of specific functional proteins, thus complementing transcriptional and post-transcriptional control mechanisms. Because of their important cellular functions, human proteases are considered as promising therapeutic targets (Bota and Davies, 2016) and their bacterial counterparts as potential antimicrobial drug targets (Culp and Wright, 2016). Hence, extending the knowledge of the substrate pools of specific proteases and the mechanisms underlying substrate selection is vital.

In prokaryotes and in the mitochondria and chloroplasts of eukaryotes, the majority of proteins is degraded by ATP-dependent proteases of the AAA+ (ATPases associated with various cellular activities) protein family (Sauer and Baker, 2011). The protease Lon was the first ATP-dependent protease to be identified and is widely conserved across the three domains of life (Gur, 2013). Lon forms a hexameric protease complex, of which each monomer contains three functional domains: an $\mathrm{N}$-terminal domain, an ATP-dependent unfoldase domain, and a peptidase domain forming the proteolytic chamber (Gur, 2013). As a heat shock protein (Phillips et al., 1984), Lon is upregulated in response to protein unfolding stress, such as thermal stress, and contributes to the degradation of unfolded and misfolded proteins that accumulate under these conditions (Gur, 2013; Gur and Sauer, 2008; Tomoyasu et al., 2001). In addition to its role in protein quality control, Lon exerts important 
regulatory functions that can be traced to the degradation of specific sets of native substrate proteins involved in stress responses, metabolism, pathogenicity, and cell cycle progression (Tsilibaris et al., 2006).

Despite many years of research on Lon proteases, the number of validated Lon substrates is small in most organisms. Most knowledge about Lon has been obtained by studying bacterial Lon orthologs. In Escherichia coli, Lon specifically degrades several stress-induced regulators (Griffith et al., 2004; Langklotz and Narberhaus, 2011; Mizusawa and Gottesman, 1983), metabolic enzymes (Arends et al., 2018) as well as antitoxins of toxin-antitoxin systems (Muthuramalingam et al., 2016), and in several pathogenic bacteria, Lon was shown to degrade regulators of pathogenicity (Joshi et al., 2020; Puri and Karzai, 2017), thus playing important roles in the regulation of virulence pathways. In the alpha-proteobacterium Caulobacter crescentus, the number of identified Lon substrates to date is small, however, Lon is known to impact cell cycle progression by degrading essential cell cycle regulators.

The $C$. crescentus cell cycle is characterized by an asymmetric cell division event and morphologically distinct cell cycle phases (Curtis and Brun, 2010). Each division yields a flagellated and piliated swarmer cell and a sessile stalked cell. While the daughter stalked cell initiates DNA replication immediately after cell division, the daughter swarmer cell must differentiate into a stalked cell before entering S-phase. Faithful progression through the $C$. crescentus cell cycle relies on precise coordination of the polar differentiation events that trigger flagella, pili, and stalk biosynthesis with core cell cycle events, such as DNA replication and cell division (Curtis and Brun, 2010). Previous work established that around one-third of all genes in C. crescentus show cell cycle-dependent fluctuations in their expression levels (Fang et al., 2013; Laub et al., 2000). Many of the corresponding proteins have important developmental functions and peak in abundance in the cell cycle phase in which their function is most needed (Laub et al., 2000). In addition to transcriptional regulatory mechanisms, active proteolysis must occur to rapidly adjust protein concentrations to enforce these transcriptional changes (Grünenfelder et al., 2001). However, only a relatively small subset of cell cycle-regulated factors with developmental functions has so far been found to be subject to proteolysis in C. crescentus and the contributions of distinct proteases in this process remain incompletely defined.

Previous work established that the ClpP protease with its unfoldases ClpA and ClpX has key roles in C. crescentus development by mediating the temporally and spatially controlled degradation of several important cell cycle regulators (Joshi and Chien, 2016; Schroeder and Jonas, 2021). In addition to ClpXP, Lon plays an important role in C. crescentus cell cycle regulation (Joshi and Chien, 2016; Schroeder and Jonas, 2021). It degrades the swarmer cell-specific transcription factor SciP (Gora et al., 2013), the methyltransferase and transcriptional regulator CcrM (Wright et al., 1996), and the conserved replication initiator DnaA (Jonas et al., 2013). Lon-dependent degradation of SciP and CcrM contributes to their cell cycle-dependent regulation (Gora et al., 2013; Wright et al., 1996), while DnaA degradation by Lon ensures rapid clearance of the protein at the onset of nutritional and proteotoxic stress, preventing cell cycle progression under these conditions (Felletti et al., 2019; Jonas et al., 2013; Leslie et al., 2015). Although $C$. crescentus cells lacking Lon are viable, they grow more slowly and show aberrant chromosome content and division defects (Leslie et al., 2015; Wright et al., 1996), which can in part be attributed to the stabilization of DnaA, SciP, and CcrM. Interestingly, $\Delta$ lon cells exhibit also characteristic developmental defects, that is, elongated stalks and motility defects (Wright et al., 1996; Yang et al., 2018), suggesting that Lon degrades additional substrates involved in cell differentiation.

Here, using a quantitative proteomics approach, we identified several proteins involved in the dimorphic life cycle of $C$. crescentus as novel Lon substrates. We studied in detail the transcriptional regulator of stalk biogenesis, StaR, and the flagella hook length regulator FliK as specific Lon substrates. We show that Lon is required to establish cell cycle-dependent fluctuations of these regulatory proteins, thereby contributing to their precise temporal accumulation during the cell cycle phase in which their function is needed. Furthermore, we demonstrate that the increased abundance of these proteins results in aberrant stalk length and motility defects. Taken together, our work revealed a critical role of Lon in coordinating cell differentiation with core cell cycle events in C. crescentus. 


\section{Results}

\section{A quantitative proteomics approach identifies novel putative Lon substrates involved in Caulobacter development}

Previous work established that Lon degrades the cell cycle regulators DnaA, CcrM, and SciP in C. crescentus (Gora et al., 2013; Jonas et al., 2013; Wright et al., 1996). Absence of Lon, either in a $\triangle$ lon strain or following Lon depletion, results in increased stability and abundance of these proteins (Figure 1A-B). Conversely, lon overexpression leads to lower protein abundance of DnaA, CcrM, and SciP (Figure 1C). Based on these results, we reasoned that it should be possible to identify novel Lon substrates by monitoring proteome-wide differences in protein stability and protein abundance in strains lacking or overexpressing lon in comparison to wild-type (WT) cells. Thus, we sampled cells from the following strain backgrounds and conditions for quantitative proteomics analysis: (1) 0,15 , and 30 min following protein synthesis shut down in the WT to assess protein stability in the presence of Lon; (2) 0,15 , and 30 min following protein synthesis shut down in $\Delta$ lon cells to examine protein stability in the absence of Lon; (3) before and after $4.5 \mathrm{hr}$ of Lon depletion in a $P_{\mathrm{van}}$-dependent Lon depletion strain; and (4) before and after $1 \mathrm{hr}$ of inducing lon overexpression from a medium-copy plasmid. Tandem mass tag (TMT) labeling and mass spectrometrical (MS) analysis were used to detect proteome-wide differences in protein levels across these samples. We obtained signals for 2270 or 2261 proteins, respectively, in our two biological replicates and sorted the proteins with respect to four criteria (see Materials and methods for details): (1) to be more stable in $\Delta$ lon cells than in the WT after 30 min of translation inhibition, (2) to be present in higher abundance at $t=0$ in $\Delta$ lon cells compared to the WT, (3) to be upregulated after Lon depletion compared to non-depleting conditions, and (4) to be downregulated following xylose-induced lon overexpression compared to noninducing conditions (Figure 1D). We identified 26 proteins that fulfilled all four criteria, one of them being DnaA. 120 proteins fulfilled three criteria, and because CcrM and SciP were in this group of proteins (Figure 1D), we considered proteins satisfying either three or four criteria as putative Lon substrates. The 146 proteins that fell into this group contained proteins from all major functional categories (Figure 1E). In comparison to all detected proteins, the group of putative Lon substrates contained a lower proportion of metabolic proteins and proteins involved in core genetic information processing, whereas proteins involved in cell cycle and cell differentiation processes, signal transduction and stress responses as well as unclassified proteins showed a higher relative abundance (Figure 1E). Notably, we did not detect FixT and HipB2 in our proteomics experiment, two other recently reported Lon substrates in C. crescentus (Stein et alo, 2020; Zhou et al., 2021).

Since $\Delta$ lon cells have previously been shown to exhibit developmental defects (Wright et alı, 1996; Yang et al., 2018), we focused this study on the group of potential Lon substrates annotated to have functions in cell cycle and cell differentiation processes. This group included proteins involved in central cell cycle regulation, chromosome partitioning, stalk morphogenesis as well as motility and chemotaxis (Figure 1F). Interestingly, according to previously published RNA-sequencing data, a large subset of the genes encoding these proteins are subject to cell cycle-dependent regulation and peak in their expression during a specific cell cycle phase (Figure 1F; Lasker et al., 2016; Schrader et al., 2016).

\section{The developmental regulator StaR is a specific Lon substrate}

One of the proteins that satisfied the four criteria in our proteomics experiment most clearly was the transcriptional regulator $\mathrm{StaR}$, a protein previously reported to be involved in the regulation of stalk biogenesis and holdfast production (Figure 1D and F; Biondi et al., 2006; Fiebig et al., 2014). Like the three known substrates DnaA, CcrM, and SciP, StaR was more stable in the $\Delta$ lon strain compared to the WT and showed increased steady-state levels in $\Delta$ lon and Lon-depleted cells, but reduced protein levels in cells overexpressing lon (Figure 2A). To validate these proteomics data, we monitored the stability of StaR in WT and $\Delta$ lon cells using a StaR-specific antibody (Fiebig et alo, 2014). In the WT, the levels of StaR were below the limit of detection, however, we observed a strong and stable band corresponding to StaR in the $\Delta$ lon strain (Figure 2B), indicating that StaR is upregulated in $\Delta$ lon cells, a result that is consistent with the proteomics data (Figure 2A). To analyze the rate of StaR degradation in the presence of Lon, we expressed staR from a medium-copy vector to elevate its levels. In this strain, StaR was degraded with a half-life of approximately $12 \mathrm{~min}$, when Lon was present (Figure 2C). Absence of Lon resulted again in complete stabilization of StaR and increased 
A

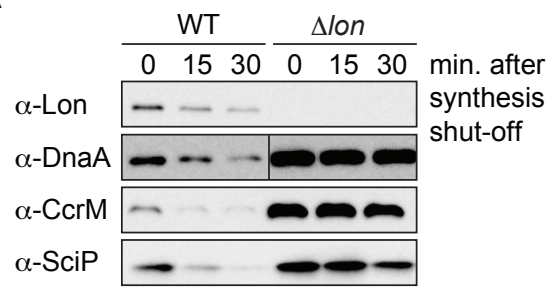

B

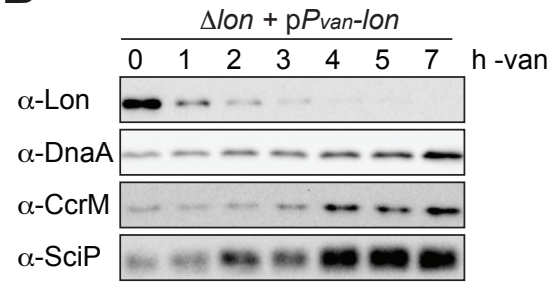

C WT

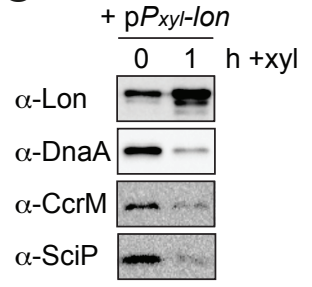

D

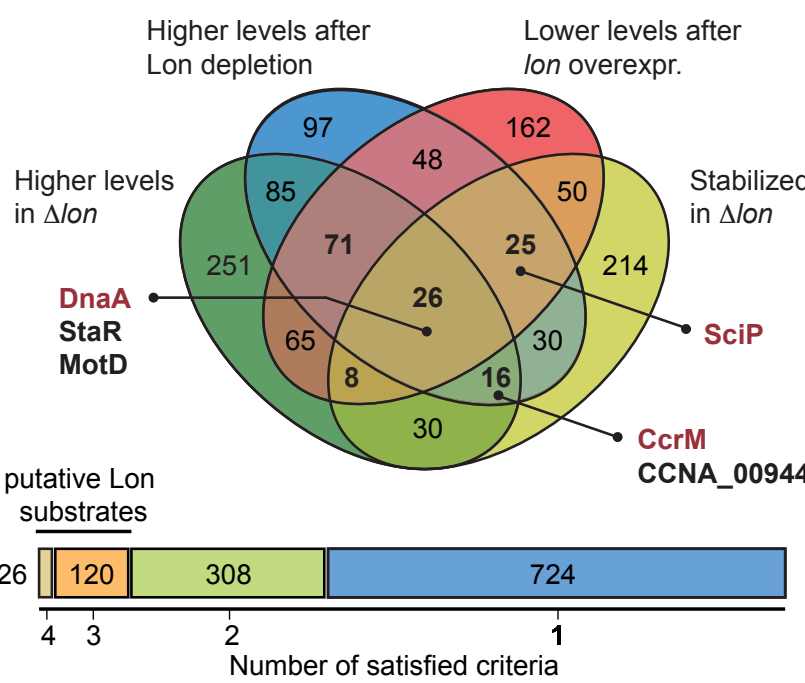

E

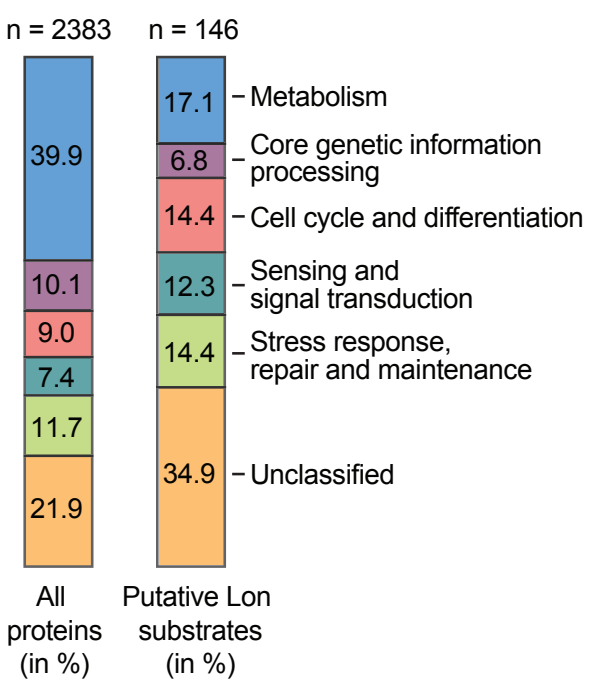

Transcript. activation

Cell cycle and polarity

\begin{tabular}{|l|l|l|l|c|}
\hline *CNA_00008 & DnaA & Chromosomal replication initiator protein & Cell cycle regulation and polarity determination & SW to ST \\
\hline *CNA_00948 & SciP & CtrA inhibitory protein SciP & Cell cycle regulation and polarity determination & SW, PD \\
\hline CCNA_00382 & CcrM & Modification methylase CcrMI & Cell cycle regulation and polarity determination & PD \\
\hline CCNA_02328 & GrA & Cell cycle regulatory protein GcrA & Cell cycle regulation and polarity determination & ST \\
\hline CCNA_01116 & DivJ & Histidine protein kinase DivJ & Cell cycle regulation and polarity determination & SW to ST \\
\hline CCNA_03584 & ChpT & Histidine phosphtransferase ChpT & Cell cycle regulation and polarity determination & ST \\
\hline CCNA_02083 & ScpB & Segregation and condensation protein B & Chromosome partitioning & - \\
\hline CCNA_03868 & ParB & Chromosome-partitioning protein ParB & Chromosome partitioning & - \\
\hline CCNA_00349 & XerC & Tyrosine recombinase XerC & Chromosome partitioning & - \\
\hline CCNA_02334 & StaR & Transcriptional regulator of stalk biogenesis & Stalk morphogenesis & SW \\
\hline CCNA_01171 & ShpA & Histidine phosphtransferase ShpA & Stalk morphogenesis & - \\
\hline CCNA_02361 & & Polysaccharide biosynthesis protein CelD & Cell envelope & SW \\
\hline CCNA_00667 & - & Lipopolysaccharide biosynthesis protein & Cell envelope & - \\
\hline
\end{tabular}

Motility and Chemotaxis

\begin{tabular}{|l|c|l|l|c|}
\hline CCNA_00945 & MotD & Chemotaxis protein MotD & Flagellum & PD \\
\hline CCNA_00944 & - & Flagellar hook length determination protein & Flagellum & PD \\
\hline CCNA_02257 & - & FliN family protein & Flagellum & PD \\
\hline CCNA_01532 & FlaY & Regulatory protein FlaY & Flagellum & SW, PD \\
\hline CCNA_00447 & CheD & Probable chemoreceptor glutamine deamidase & Chemotaxis & PD \\
\hline CCNA_02976 & - & MCP-signal associated domain protein & Chemotaxis & SW, PD \\
\hline CCNA_03247 & - & Methyl-accepting chemotaxis protein & Chemotaxis & PD \\
\hline CCNA_03257 & - & Chemotaxis receiver domain protein CheYIII & Chemotaxis & - \\
\hline
\end{tabular}

Figure 1. A quantitative proteomics approach identifies putative Lon substrates in Caulobacter crescentus. (A) In vivo stability assays of Lon, DnaA, CcrM, and SciP in the wild-type (WT) and the $\Delta$ lon strain (LS2382). Protein synthesis was shut down by addition of chloramphenicol at $t=0$ and remaining protein levels were measured after 15 and $30 \mathrm{~min}$. The protein levels at $\mathrm{t}=0$ correspond to the steady-state levels of these proteins in the WT and the $\triangle$ Ion (LS2382) mutant. (B) Protein levels of Lon, DnaA, CcrM, and SciP over $7 \mathrm{hr}$ of Lon depletion. The expression of lon was shut off by transferring the $P_{\text {van }}$-dependent Lon depletion strain (ML2022) from PYE supplemented with vanillate (van) to PYE lacking vanillate (-van). (C) Protein levels of Lon, DnaA, CcrM, and SciP in a $P_{x y l}$-dependent lon overexpression strain (ML2010) before (0) and $1 \mathrm{hr}$ after induction with xylose (1 hr+xyl). (D) Venn chart showing groups of proteins that meet the following criteria and how they overlap: (1) to be present in higher abundance at steady state in $\Delta$ lon cells compared to the WT (green circle), (2) to be upregulated after $4.5 \mathrm{hr}$ of Lon depletion compared to non-depleting conditions (blue circle), (3) to be downregulated Figure 1 continued on next page 
Figure 1 continued

after $1 \mathrm{hr}$ of induced lon overexpression compared to non-inducing conditions (red circle), and (4) to be stabilized in $\Delta$ lon cells compared to the WT $30 \mathrm{~min}$ after translation shut off (yellow circle). Previously confirmed Lon substrates (shown in red) and the proteins investigated in this study (shown in black) are highlighted. The bar graph below the Venn chart indicates the number of proteins satisfying four, three, two, or one criteria. Proteins satisfying either three or four criteria were considered as putative Lon substrates. (E) Putative Lon substrates identified in (D) sorted by functional category (right bar graph). For comparison, the sorting of all detected proteins into functional categories is shown (left bar graph). (F) Tables listing the putative Lon substrates with functions in cell cycle and polarity as well as motility and chemotaxis. Previously known Lon substrates are marked with an asterisk. Proteins satisfying four criteria are highlighted in green. The cell cycle phase in which the expression of the listed proteins is transcriptionally induced is indicated (SW: swarmer cell, ST: stalked cell, PD: predivisional cell, SW to ST: swarmer to stalked cell transition).

The online version of this article includes the following figure supplement(s) for figure 1 :

Source data 1. Proteomics data.

Source data 2. Unprocessed Western blot images.

steady-state levels, which is in line with our proteomics data, and confirms that StaR degradation depends on Lon. To directly test if StaR is a Lon substrate, we performed an in vitro degradation assay with purified StaR and Lon. This assay showed that Lon readily degrades StaR in an ATP-dependent manner (Figure 2D). Hence, StaR is a Lon substrate and no additional factors are required for recognizing and degrading StaR, at least in vitro.

We also investigated if Lon recognizes StaR via a degron sequence at one of the termini and monitored the degradation of FLAG-tagged StaR variants. Addition of the 3xFLAG tag to the C-terminus of StaR (StaR-F) completely abolished degradation (Figure 2E), indicating that a freely accessible C-terminus of StaR is required for degradation by Lon. Addition of the tag to the N-terminus of StaR (F-StaR) still enabled notable degradation within $60 \mathrm{~min}$ after shutting down protein synthesis (Figure 2E). However, degradation of this $\mathrm{N}$-terminally tagged StaR was also observed in the $\Delta$ lon strain, suggesting that another protease degrades this StaR variant, probably because of changes in StaR folding that result from the addition of the tag. These data show that native $\mathrm{N}$-termini and $\mathrm{C}$-termini of the protein are required for Lon-dependent degradation.

\section{Lon ensures cell cycle-dependent regulation of StaR abundance}

As a transcriptional regulator of stalk biogenesis and holdfast production, StaR function is expected to be particularly needed at the beginning of the cell cycle, when the swarmer cell differentiates into a stalked cell (Figure 3A). Indeed, previously published RNA sequencing data show that staR mRNA levels fluctuate during the cell cycle, peaking in the swarmer and early stalked cell before declining during S-phase and remaining low until cell division (Figure 3B; Lasker et al., 2016; Schrader et al., 2016). Furthermore, existing ribosome profiling data show that StaR is translated predominantly in the swarmer and early stalked cells, but only at low levels during the remaining cell cycle (Lasker et al., 2016; Schrader et al., 2016). Quantification of StaR protein levels by Western blot analysis in synchronized cultures showed that protein levels follow this expression pattern in WT cultures (Figure 3C). StaR was detectable within 30 min after synchronization before it was strongly downregulated and remained below the limit of detection for the rest of the cell cycle. Strikingly, the cell cycle-dependent changes in protein abundance were absent in the $\Delta$ lon strain, in which StaR levels remained high until 75 min after synchronization (Figure 3C). Thus, Lon-dependent degradation of StaR is required for establishing oscillations of StaR levels during the cell cycle.

\section{Lon-mediated StaR proteolysis is required for proper stalk biogenesis}

Next, we assessed the importance of Lon-mediated StaR proteolysis for correct stalk biogenesis. Consistent with a previous study (Wright et al., 1996), we observed that the stalks of $\Delta$ lon cells are significantly elongated compared to the WT (Figure 3D-E). Because StaR overexpression was shown to lead to an increase of stalk length (Biondi et al., 2006), we reasoned that the abnormal stalk length of $\Delta$ lon cells might be caused by the higher abundance of StaR in these cells. To address this hypothesis, we introduced the $\Delta s t a R$ deletion into the $\Delta$ lon strain background and assessed stalk length of this $\Delta$ staR $\Delta$ lon double mutant (Figure 3D-E, Figure 3-figure supplement 1). The $\Delta$ staR $\Delta$ lon mutant phenocopied the $\Delta$ staR single mutant, in which stalks are shortened compared to $\Delta$ lon cells, demonstrating that Lon affects stalk length through StaR (Figure 3D-E). To further investigate the relationship between Lon, StaR, and stalk lengths, we also analyzed stalk morphology under 
A

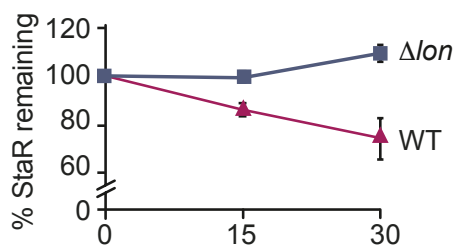

min. after synthesis shut-off

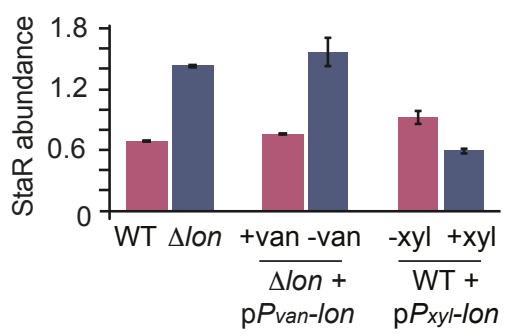

B

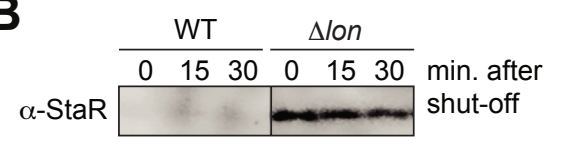

C

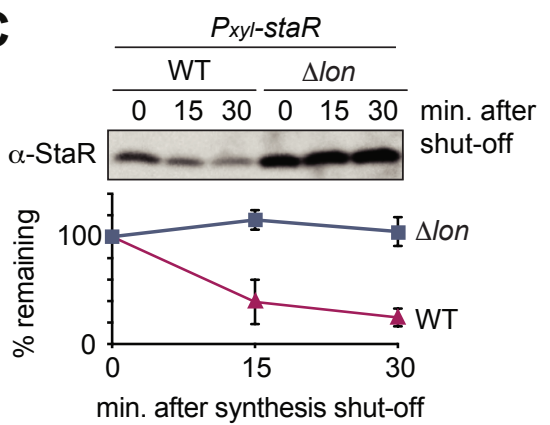

D

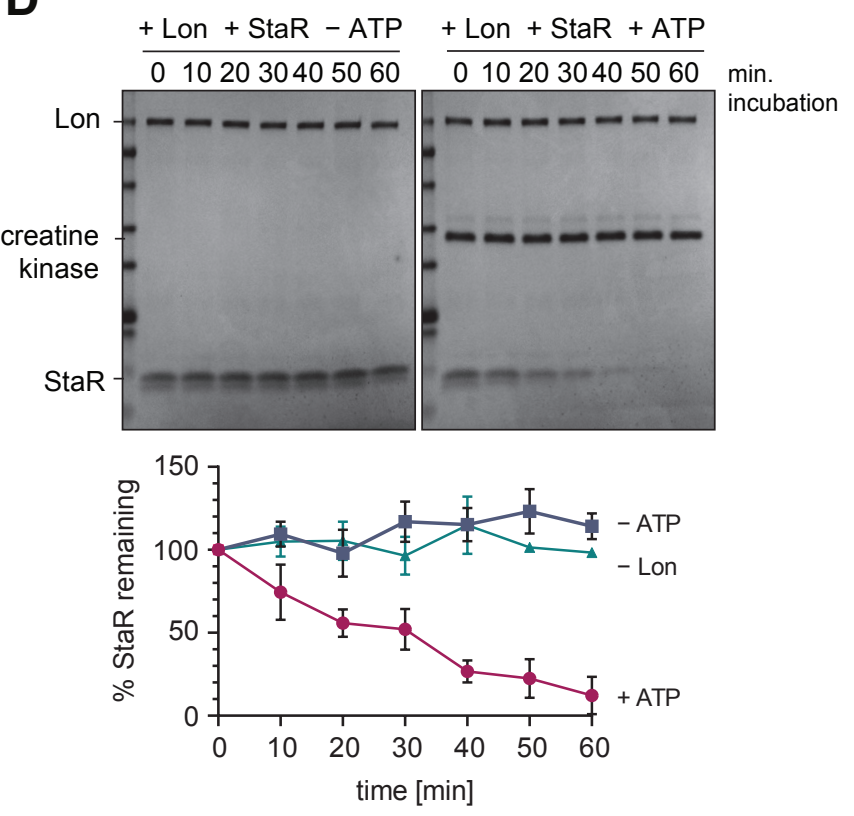

E

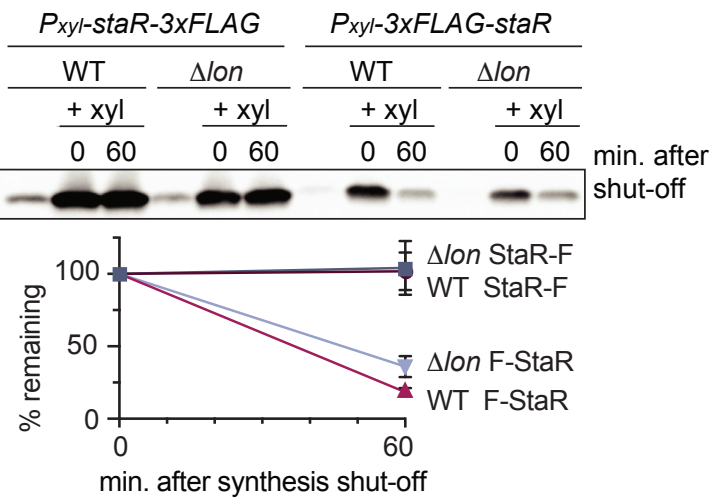

Figure 2. The developmental regulator StaR is a Lon substrate. (A) Proteomics data obtained for StaR. The upper graph shows StaR stability in wildtype (WT) and $\Delta$ lon (LS2382) cells and the lower graph StaR abundance in the different strain backgrounds and conditions as determined by mass spectrometry. Each data point represents the mean protein abundance of the two experimental replicates, error bars show standard deviations. (B) In vivo degradation assay of StaR at native expression levels in WT and $\Delta$ lon (KJ546) cells. (C) In vivo degradation assay of StaR after xylose-induced staR overexpression in WT and $\Delta$ lon (KJ546) backgrounds. The graph shows mean values and standard deviations of relative protein levels after protein synthesis shut off determined from three independent experiments. (D) In vitro assay showing degradation of StaR by Lon. $4 \mu \mathrm{M}$ StaR and $0.125 \mu \mathrm{M}$ Lon hexamer was incubated in the presence (+ATP) or absence (-ATP, control) of an ATP regeneration system. The graph shows the relative StaR levels normalized to Lon or CK (in case of the -Lon sample) levels of three independent experiments and are represented as means with standard deviations. (E) N-terminally and C-terminally tagged StaR (3xFLAG-StaR and StaR-3xFLAG, respectively) were expressed ectopically by xylose induction and their protein levels were assessed in WT and $\Delta$ lon (KJ546) cells prior to induction (first lanes of each set of three) and after induction either before ( $t=0$ min) or after shutting off protein synthesis ( $\mathrm{t}=60 \mathrm{~min}$ ). The graph shows the mean values of relative protein levels and standard deviations of three independent experiments.

The online version of this article includes the following figure supplement(s) for figure 2:

Source data 1. Unprocessed Western blot and protein gel images.

phosphate-limiting conditions, in which stalks are drastically elongated in C. crescentus (Schmidt and Stanier, 1966). Stalk length in the different strain backgrounds followed the same trend as under optimal conditions (Figure 3-figure supplement 1). However, all four strains were able to strongly elongate their stalks under phosphate starvation, suggesting that StaR and Lon are not required 
A

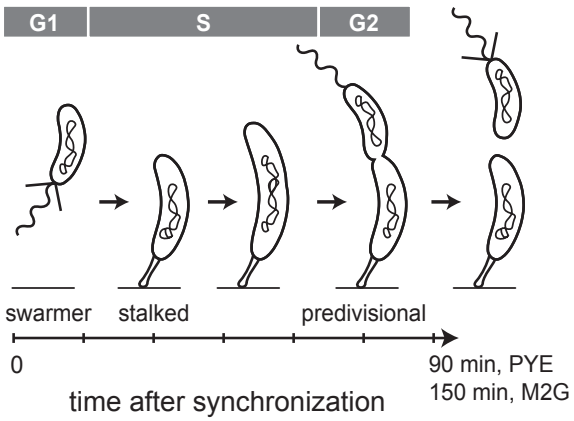

B

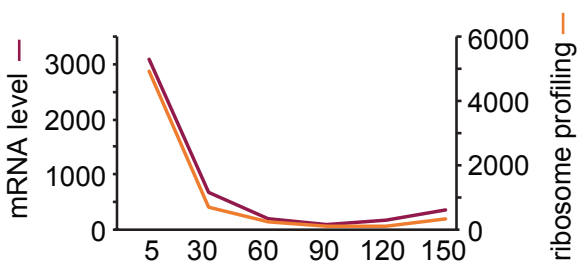

min. after synchronization (M2G)

C

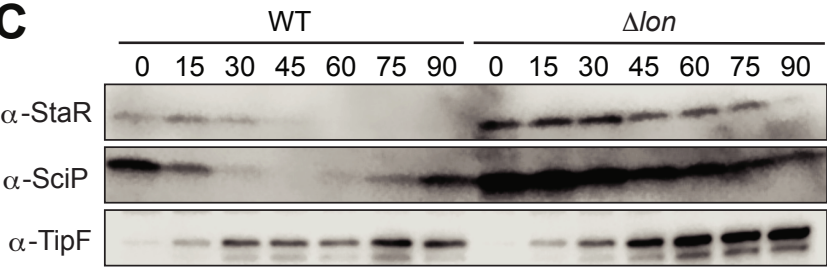

D
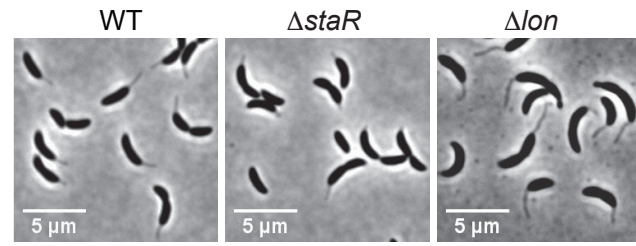

$\Delta s t a R \Delta / o n$

min. after synchrony (PYE)

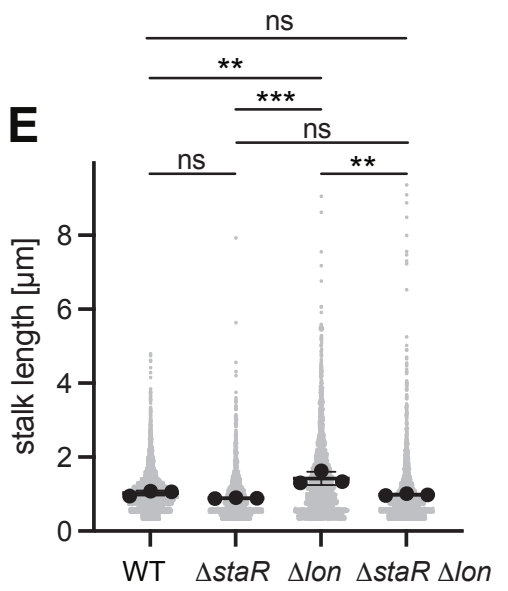

Figure 3. Lon ensures cell cycle-dependent accumulation of StaR and proper stalk length regulation. (A) Schematic illustration of the Caulobacter crescentus cell cycle and how the different cell cycle phases are associated with distinct morphological states. The time line indicates how the cell cycle progresses over time. (B) RNA-sequencing and ribosome profiling data for staR, as published previously (Lasker et al., 2016; Schrader et al., 2016). (C) Protein levels of StaR, SciP, and TipF in synchronized wild-type (WT) and $\Delta$ lon (KJ546) culture over 90 min following release of swarmer cells into PYE medium. Western blots for SciP and TipF were included as controls for proteins with well-characterized cell cycle patterns (Davis et al., 2013; Gora et al., 2010). (D) Phase contrast microscopy images depicting morphological differences between $C$. crescentus WT the single mutants $\Delta$ staR and $\Delta$ lon (KJ546) and the double mutant $\triangle$ staR $\triangle$ lon when grown in PYE at $30^{\circ} \mathrm{C}$. (E) Quantifications of stalk length under optimal conditions (PYE, $30^{\circ} \mathrm{C}$ ) of the strains shown in (D). N was at least 1800 total for each strain obtained from three biological replicates. Statistical significance was determined by ordinary one-way ANOVA (Tukey's multiple comparisons test: WT vs. $\Delta$ lon $p=0.0047,{ }^{\star *} ; \Delta$ staR vs. $\Delta$ lon $p=0.0007,{ }^{\star * *} ; \Delta$ lon vs. $\Delta$ staR $\Delta$ lon $p=0.0025,{ }^{*}$; WT vs. $\Delta$ staR $\mathrm{p}=0.3591$, not significant; $\Delta$ staR vs. $\Delta$ staR $\Delta$ lon $\mathrm{p}=0.6398$, not significant; WT vs. $\Delta$ staR $\Delta$ lon $\mathrm{p}=0.9447$, not significant).

The online version of this article includes the following figure supplement(s) for figure 3 :

Source data 1. Unprocessed Western blot images.

Figure supplement 1. Quantification of stalk length under standard (M2G) and phosphate-limiting conditions (M5G).

for starvation-dependent stalk elongation. Taken together, our data demonstrate that Lon-mediated degradation of StaR is required for proper stalk biogenesis during the cell cycle.

\section{The two putative Lon substrates CCNA_00944 and MotD are part of one single protein that corresponds to FliK}

In addition to StaR, our proteomics experiments identified several proteins involved in flagellabased motility and chemotaxis as potential Lon substrates (Figure 1F). Particularly promising hits in this group of proteins were CCNA_00944 and MotD (CCNA_00945) that are encoded by partly overlapping open reading frames and showed similar changes in protein abundance and stability in the Lon deficient and Lon overproducing strains in our proteomics experiments (Figure 4A-B). While CCNA_00944 is annotated as a flagella hook length determination protein, MotD is annotated as a chemotaxis protein. According to a signature-based annotation of MotD in UniProtKB (entry 
A

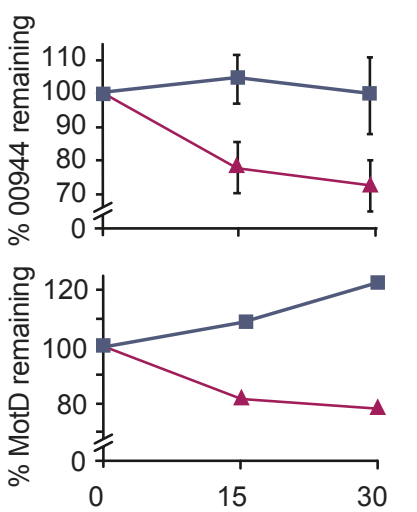

min. after synthesis shut-off

D

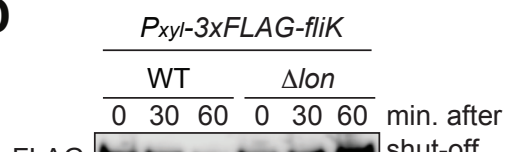
$\alpha$-FLAG shut-off

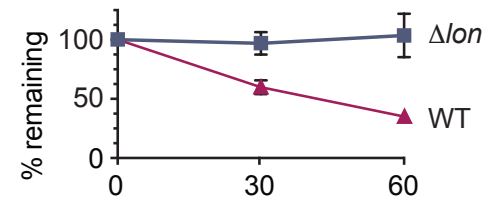

min. after synthesis shut-off

F

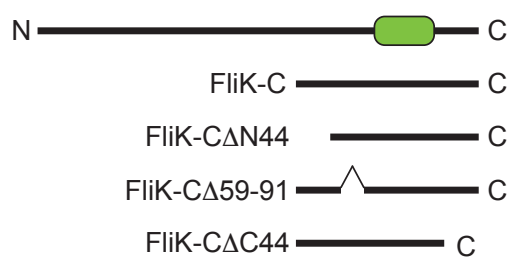

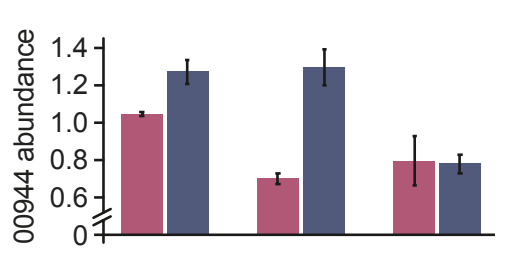

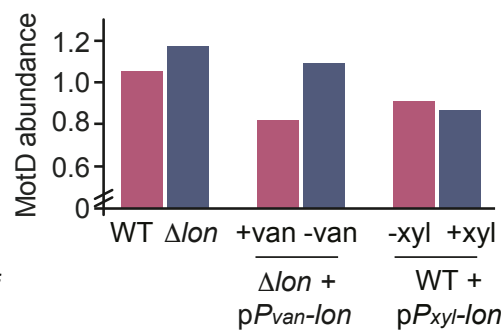

E Pxy-3xFLAG-flik-C WT $\Delta / 0$ n

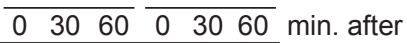
$\alpha$-FLAG $\backsim-\infty \sim \infty \sim$ shut-off

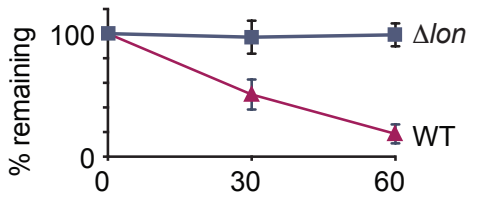

min. after synthesis shut-off
B
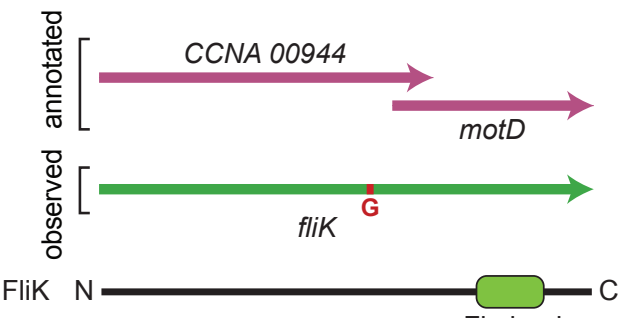

Flg hook
C

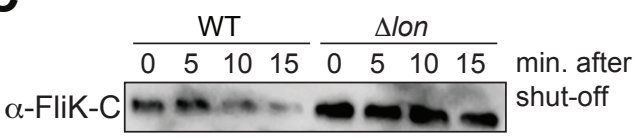

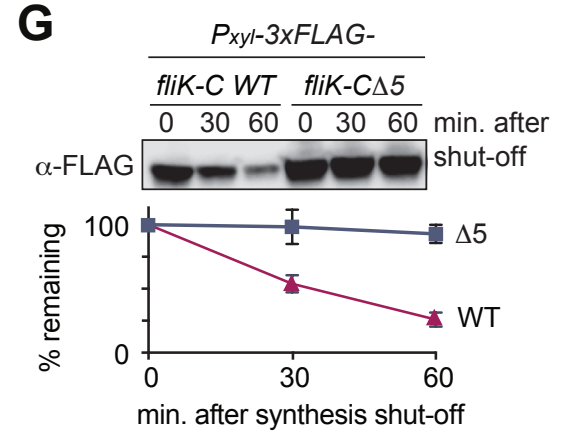

min. after synthesis shut-off

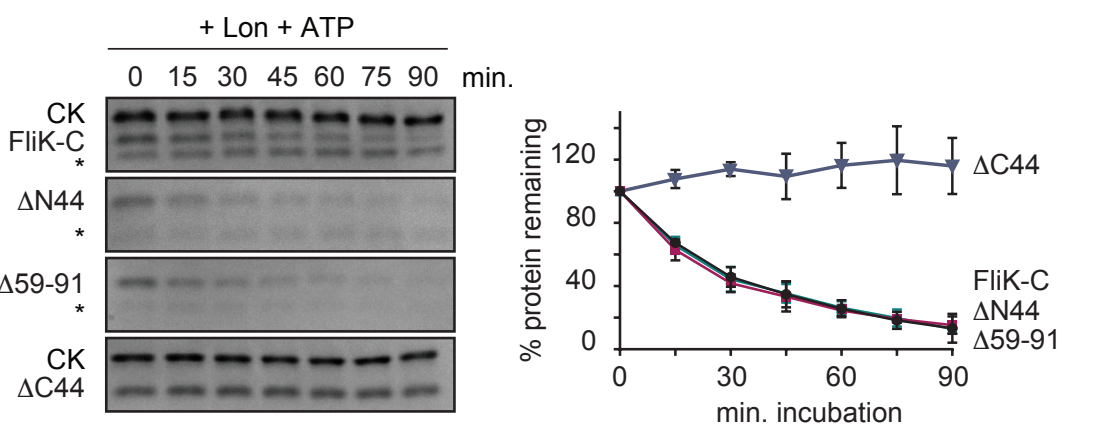

Figure 4. The flagella hook length regulator FliK is a Lon substrate with a C-terminal degradation tag. (A) Proteomics data obtained for CCNA_00944 and CCNA_00945 (MotD). The graphs on the left show CCNA_00944 and MotD stability in wild-type (WT) and $\triangle$ lon (LS2382) cells, and graphs on the right show CCNA_00944 and MotD abundance in the different strain backgrounds and conditions as determined by mass spectrometry. Each data point for CCNA_00944 represents the mean protein abundance of the two experimental replicates, error bars show standard deviations. MotD was only detected in one of the replicates. (B) Schematic representation of the CCNA_00944 and motD (CCNA_00945) genes as annotated in the Caulobacter crescentus NA1000 genome, and how the presence of an additional guanosine (highlighted in red) merges the two genes to form one continuous gene that was named fliK. The corresponding FliK protein contains a Flg hook domain. (C) In vivo degradation assay of full-length FliK in WT and $\triangle$ lon (KJ546) cells. Samples were taken 0, 5, 10, and 15 min after shutting off protein synthesis. (D) In vivo degradation assay of ectopically expressed $\mathrm{N}$-terminally 3xFLAG-tagged full-length FliK in WT and $\Delta$ lon (KJ546) cells. The graph shows mean values and standard deviation of relative protein levels 0,30 , and 60 min after protein synthesis shut off determined from three independent experiments. (E) In vivo degradation assay of the C-terminal part of FliK (FliK-C) in WT and $\triangle$ lon (KJ546) cells. N-terminally 3xFLAG tagged FliK-C was ectopically expressed and samples were taken at indicated time points after shut off of protein synthesis. Quantifications show mean values obtained from three independent biological replicates and error bars represent standard deviation. (F) In vitro degradation assays showing Lon-dependent degradation of FliK-C and truncated FliK-C proteins lacking N-terminal, internal, or C-terminal regions, graphically illustrated on the left side of the panel. Degradation assays were carried out in Lon reaction buffer with $4 \mu \mathrm{M}$ of one of the FliK-C variants, $0.125 \mu \mathrm{M}$ of Lon hexamer in the presence of the ATP regeneration system (ATP, creatine phosphate, and creatine kinase $[C K])$. Asterisks $\left(^{\star}\right)$ mark a low molecular weight protein, that co-purifies with FliK-C (and some variants) but is unaffected by Lon. The band intensities

Figure 4 continued on next page 
Figure 4 continued

of the FliK-C variants from three independent experiments are represented as means with standard deviations on the right side of the panel. (G) In vivo degradation assay of N-terminally 3xFLAG tagged FliK-C (WT) and a variant lacking the C-terminal five amino acids (FliK-C $\triangle 5$ ) after xylose induction in WT cells. Samples were taken at indicated time points after shut off of protein synthesis. Quantifications show mean values of relative protein levels obtained from three biological replicates and error bars represent standard deviation.

The online version of this article includes the following figure supplement(s) for figure 4 :

Source data 1. Unprocessed Western blot and protein gel images.

Figure supplement 1. Western blots of native FliK and 3xFLAG tagged FliK.

Figure supplement 1-source data 1. Unprocessed Western blot images.

Figure supplement 2. In vitro FliK-C degradation by Lon is ATP-dependent.

Figure supplement 2-source data 1. Unprocessed protein gel image.

AOAOH3C6R3), MotD contains a conserved Flg hook domain that is commonly present in the C-terminal portion of FliK proteins that control flagella hook length in many bacteria (Waters et al., 2007). When we attempted to clone CCNA_00944, we repeatedly observed one additional guanosine in the cloned gene sequence that was not present in the reference genome sequence of $C$. crescentus NA1000, the strain that we use. This insertion, which is also present in the sequence reads of previously published RNA-sequencing data (Schrader et al., 2014), generates a frameshift that merges the CCNA_00944 gene with the downstream located motD gene, thus forming one single open reading frame, of which the $3^{\prime}$ portion corresponds to motD (Figure 4B). This resulting gene corresponds to a single open reading frame (CC_0900) in C. crescentus CB15, the isolate from which NA1000 is derived. These observations indicate that CCNA_00944 and motD are incorrectly annotated in the reference genome of NA1000 and instead form one continuous open reading frame. Consistently, when we performed Western blot analysis with antiserum raised against the protein portion corresponding to MotD, we detected one single protein band that runs at high molecular weight (Figure 4-figure supplement 1), confirming that CCNA_00944 and motD form together one single open reading frame. Because the C-terminal portion of this new gene encodes a Flg hook domain that is a characteristic of FliK proteins in other bacteria and because no other gene has so far been annotated as fliK in C. crescentus, we named the new gene fliK (Figure 4B). This annotation of fliK is also in line with a recent study in Sinorhizobium meliloti, which suggested that the motD gene from alpha-proteobacteria should be renamed fliK (Eggenhofer et al., 2006).

\section{FliK is a Lon substrate that is recognized at its C-terminus}

Having established that CCNA_00944 and MotD are part of the same FliK protein, we next investigated its regulation by Lon. Consistent with our proteomics data, we found that FliK abundance and stability were notably increased in $\Delta$ lon cells, consistent with FliK being a Lon substrate (Figure $4 C$ ). To investigate the sequence determinants required for Lon to interact with FliK, we expressed an $\mathrm{N}$-terminally FLAG-tagged version of FliK and monitored its stability in vivo. Similar to the non-tagged FliK, 3xFLAG-FliK was efficiently degraded in WT cells but stable in cells lacking Lon (Figure 4D). This result led us to hypothesize that the recognition by Lon occurs via the C-terminal domain of FliK. Therefore, we monitored the stability of the C-terminal portion of FliK containing the Flg hook domain, which corresponds to the formerly annotated MotD protein. Like the full-length protein, degradation of this truncated FliK protein (FliK-C), with a FLAG-tag at the N-terminus, depended strongly on Lon (Figure 4E). Furthermore, in vitro degradation assays showed that Lon degrades non-tagged FliK-C in an ATP-dependent manner (Figure 4F, Figure 4-figure supplement 2). Based on these results, we conclude that FliK is a Lon substrate, and that its C-terminal portion containing the Flg hook domain is sufficient for Lon-dependent degradation.

To further pinpoint the regions within FliK-C that are required for Lon-dependent turnover, we analyzed the degradation of a set of additional truncation mutants. According to the MobiDB database (Piovesan et al., 2021), the C-terminal portion of FliK lists two unordered regions. We engineered FliK-C variants that lack either of these unordered regions (FliK-C $\Delta$ N44 and FliK-C $\Delta 59-91$ ) or the C-terminal part (FliK-C $\Delta \mathrm{C} 44)$. In vitro degradation assays showed that deletion of the unordered regions did not influence degradation, whereas removal of the $44 \mathrm{C}$-terminal amino acids completely stabilized the protein (Figure 4F). This result, and the fact that some of the degrons recognized by 
Lon are located at the very C-terminus of Lon substrates (Burgos et al., 2020; Ishii et al., 2000; Puri and Karzai, 2017; Zhou et al., 2019), prompted us to determine the in vivo stability of a FliK-C variant lacking only the C-terminal five amino acids with the sequence LDIRI (3xFLAG-FliK-C $\Delta 5$ ) (Figure 4G). This deletion abolished FliK-C degradation (Figure 4G), demonstrating that the interaction between Lon and FliK depends on these C-terminal amino acids.

\section{Lon ensures temporally restricted accumulation of FliK during the cell cycle}

Like many other proteins involved in flagella biogenesis in C. crescentus, the transcription of the two annotated genes CCNA_00944 and motD that together form the flik gene is cell cycle regulated (Lasker et al., 2016; Schrader et al., 2016), with mRNA levels and ribosome occupancy peaking in late S-phase when a new flagellum at the pole opposite the stalk is being assembled (Figure 5A). Consistently, Western blot analysis with synchronized WT C. crescentus cultures showed that FliK protein was not detectable in the beginning of the cell cycle, but began to accumulate in late stalked cells before reaching a maximum in abundance in predivisional cells shortly before cell division (Figure 5B). This cell cycle-dependent pattern of FliK abundance was completely absent in the $\Delta$ lon strain, in which FliK was already detectable in swarmer cells and remained at high levels throughout the cell cycle (Figure 5B). This result shows that, as in the case of StaR, Lon is absolutely necessary to ensure that the protein is eliminated in the cell cycle phase when its function is no longer needed.

\section{Precise regulation of FliK abundance is required for proper flagellin expression and flagella function}

Next, we wanted to investigate if the Lon-dependent regulation of FliK abundance is required for proper motility in C. crescentus. Consistent with a previous study (Yang et al., 2018), we observed that $\Delta$ lon cells show reduced motility in soft agar compared to the WT (Figure $5 \mathrm{C}$ ), which might be caused by reduced flagella function in addition to growth and cell division defects. Additionally, our proteomics data revealed that the levels of $\alpha$-flagellins FljJ, FljK, and FljL and $\beta$-flagellins FljM and FljN, which compose the structural components of flagella, are strongly downregulated in the $\Delta$ lon mutant (Figure 5D). Although a Lon-dependent effect on flagellin levels was not apparent after $4.5 \mathrm{hr}$ of Lon depletion and only to a lesser extent upon lon overexpression (Figure 5D), these data point to an indirect involvement of Lon in the regulation of flagella biosynthesis. One explanation for the motility defect and the reduced flagellin levels in the $\Delta$ lon mutant might be the stabilization and higher levels of SciP in this strain (Figure 1A; Gora et al., 2013), which is known to negatively affect flagellin gene expression through CtrA (Gora et al., 2010). Since correct regulation of FliK levels was shown to be critical for proper flagella biosynthesis in other species (Muramoto et al., 1998; Waters et al., 2007), we thought that the stabilization and thus increased abundance of FliK in the absence of Lon might contribute to the motility defect of $\Delta$ lon cells as well. To specifically study the consequences of increased FliK abundance, we overexpressed FLAG-tagged FliK, FliK-C, and FliK$\mathrm{C} \Delta 5$ from a medium copy vector in otherwise WT cells and assessed soft agar motility and flagellin levels. Overexpression of FliK led indeed to a reproducible reduction in swim diameter to $85 \%$ compared to the vector control strain (Figure 5E), indicating that elevated levels of FliK impair motility. Interestingly, while this effect was clearly exacerbated in the strain overexpressing FliK-C (Figure 5E-F), it was absent in the strain overexpressing FliK-C $\Delta 5$, demonstrating that the C-terminus of FliK is critical for the FliK-dependent effect on motility. When analyzing flagellin protein levels in the different overexpression strains, we found that the motility defects of the FliK and FliK-C overexpression strains correlated with a significant downregulation of flagellin levels to $55 \%$ and $30 \%$, respectively (Figure $5 \mathrm{G}$ ). Conversely, overexpression of the FliK-C $\Delta 5$ affected flagellin levels only mildly (Figure 5G).

Taken together, our data indicate that an oversupply of FliK, which can either be caused by overexpression or absence of Lon-dependent degradation, leads to reduced motility and flagellin levels. This suggests that FliK likely contributes to the motility defects of $\Delta$ lon cells along with SciP and potentially other Lon substrates affecting flagella function that are stabilized and upregulated in the absence of Lon. Furthermore, our data revealed that the C-terminal portion of FliK is critical not only for FliK degradation, but also for its effects on motility and flagellin protein levels. 


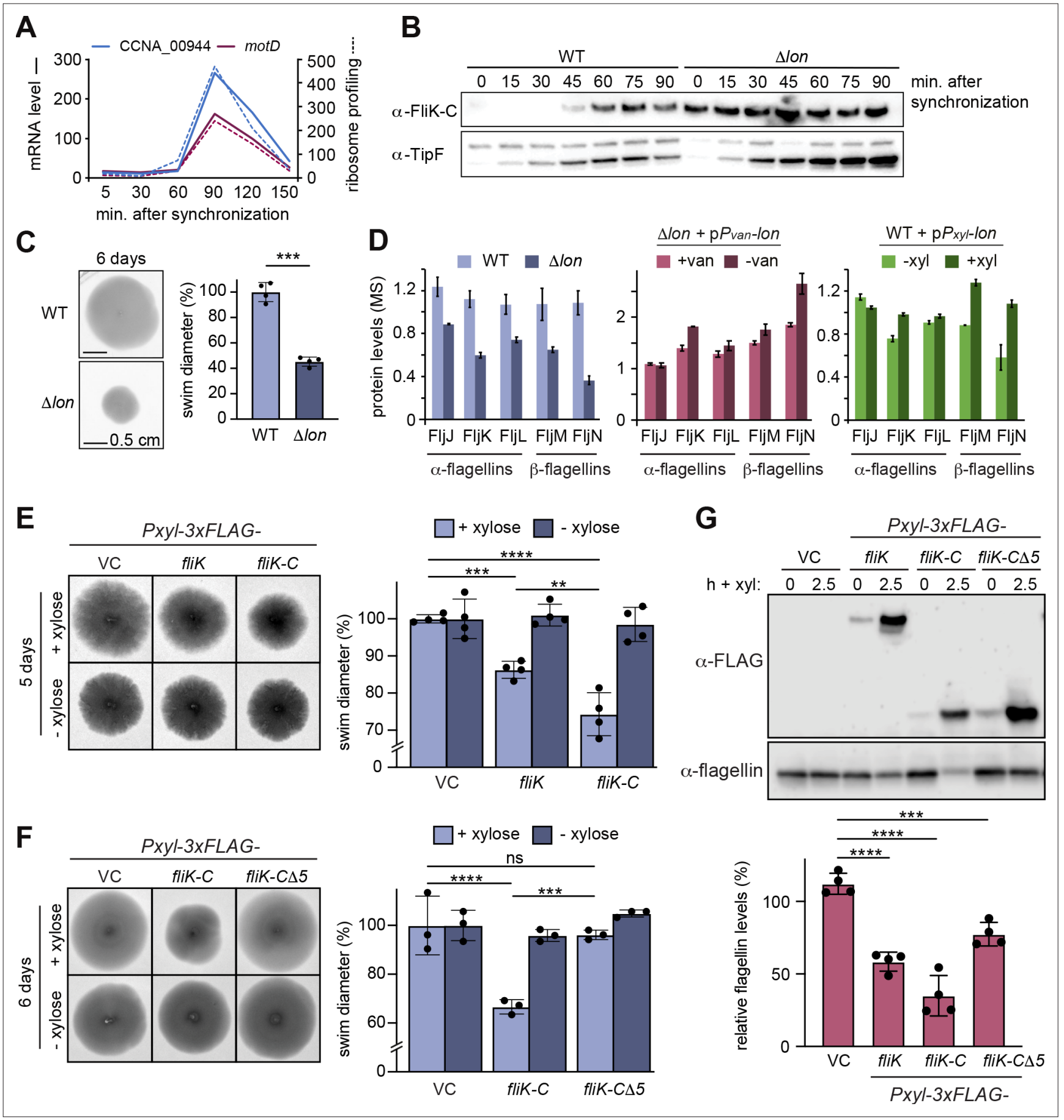

Figure 5. Lon-dependent degradation ensures temporal regulation of FliK levels during the cell cycle, which is needed for normal flagellin expression and motility. (A) RNA-sequencing and ribosome profiling data for CCNA_00944 and motD (CCNA_00945), as previously published (Lasker et al., 2016; Schrader et al., 2016). (B) Protein levels of FliK and TipF in synchronized wild-type (WT) and $\Delta$ lon (KJ546) cultures over 90 min following release of swarmer cells into PYE medium. TipF was included as a control. (C) Motility assay of Caulobacter crescentus WT and the $\triangle$ lon (KJ546) mutant in PYE soft agar after 6 days. The graph shows the relative swim diameters from four biological replicates, means (mean WT value was set to 100\%), and standard deviations are indicated. Statistical significance was determined by paired two-tailed t-test: WT vs. $\Delta$ lon $p=0.0002,{ }^{* \star *}$. (D) Flagellin protein levels as determined by mass spectrometry in WT and $\Delta$ lon (LS2382) mutant cells as well as in the other strain backgrounds and conditions, see Figure 1. (E) Motility assay in soft agar of strains overexpressing 3xFLAG-tagged FliK and FliK-C by xylose induction (+xylose) in comparison to the vector control (VC) and non-inducing conditions (-xylose). The graph shows the relative swim diameters from four biological replicates, means (mean of VC was set to 100\%) and standard deviations are indicated. Statistical significance was determined by ordinary one-way ANOVA (Šidák's multiple comparisons test)

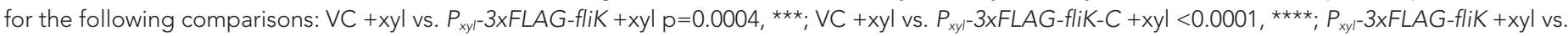
Figure 5 continued on next page 
Figure 5 continued

$P_{x y l}-3 x F L A G-f l i K-C+x y l p=0.0016$, **. (F) Motility assay in soft agar of strains overexpressing 3xFLAG-tagged FliK-C and FliK-C $\Delta 5$ by xylose induction (+xylose) in comparison to the VC and non-inducing conditions (-xylose). The graph shows the relative swim diameters from three biological replicates, means (relative to VC) and standard deviations are indicated. Statistical significance was determined by ordinary one-way ANOVA (Šidák's multiple comparisons test) for the following comparisons: VC +xyl vs. $P_{x y l}-3 x F L A G-f l i K-C+x y l p<0.0001$, ****; VC +xyl vs. $P_{x y l}-3 x F L A G-f l i K-C \Delta 5+x y l p=0.8184$, not significant; $P_{x y l}-3 x F L A G-f l i K-C+x y l$ vs. $P_{x y l}-3 x F L A G-f l i K-C \Delta 5+x y l p=0.0001, * * *$. (G) Western blot analysis showing total flagellin levels of strains harboring the empty vector (VC) or plasmids for expression of 3xFLAG-tagged FliK, FliK-C, or FliK-C 55 before (0) and after induction of expression by xylose for $2.5 \mathrm{hr}$ (lower panel). Induction of the respective FliK variants was determined by Western blot analysis using an anti-FLAG antibody (upper panel). The graph shows the relative flagellin levels compared to the uninduced condition (without xylose) for each strain, as determined by four independent experiments, mean values and standard deviations are indicated. Statistical significance was determined by ordinary one-way ANOVA (Šidák's multiple

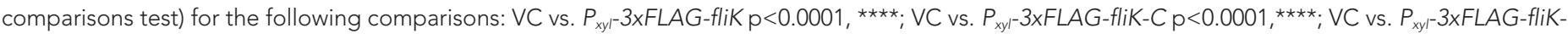
$C \triangle 5 \mathrm{p}=0.0006$, ${ }^{* * *}$.

The online version of this article includes the following figure supplement(s) for figure 5 :

Source data 1. Unprocessed Western blot images.

\section{Discussion}

In all cells, the concentrations of specific proteins must be precisely regulated to maintain cellular functions and to orchestrate complex cellular behaviors in response to external and internal cues. This study uncovered a novel role of the highly conserved protease Lon in coordinating cell differentiation with cell cycle processes in the dimorphic bacterium C. crescentus. In this bacterium, each cell cycle phase is coupled to a distinct morphological state (Curtis and Brun, 2010). This coupling of cell differentiation with core cell cycle events requires sophisticated mechanisms that coordinate these processes in space and time. Previous work established that in C. crescentus large sets of genes are transcriptionally regulated in a cell cycle-dependent manner (Laub et al., 2000; McGrath et al., 2007; Schrader et al., 2016). Using a proteomics approach, we found that several proteins encoded by cell cycle-regulated genes are Lon substrates. The identified Lon substrates include important regulators and structural components required for $C$. crescentus development and cell cycle progression. Our results show that active proteolysis of at least some of these proteins by Lon is required to rapidly clear these proteins following a cell cycle-dependent decrease in their transcription, thus restricting their accumulation to the cell cycle phase when their function is needed (Figure 6). The abundance of Lon does not change during the cell cycle (Wright et al., 1996) and a recent study suggested that the catalytic activity of Lon is cell cycle independent (Zhou et al., 2019). However, it is possible that the degradation of Lon-dependent degradation is affected by the accessibility or functional state of Lon substrates. For example, previous work showed that degradation of both SciP and CcrM is modulated by DNA binding (Gora et al., 2013; Zhou et al., 2019), and in the case of the AAA+ ATPase DnaA, ATP binding seems to increase protein stability (Liu et al., 2016; Wargachuk and Marczynski, 2015). Future work will show if similar mechanisms modulate the degradation of the herein identified Lon substrates.

We focused our studies on the stalk regulator StaR and the flagella regulator FliK, which were among the proteins whose abundance and stability were most strongly affected by Lon. Like DnaA, CcrM, and SciP, the transcriptional regulator StaR is a DNA-binding protein. It was initially identified as a positive regulator of stalk biogenesis (Biondi et al., 2006) and was later shown to regulate holdfast development by directly inhibiting the expression of the holdfast inhibitor HfiA (Fiebig et al., 2014); holdfast is a polysaccharide-rich adhesin that is produced at the nascent stalked cell pole in late swarmer cells and allows C. crescentus to attach to surfaces (Curtis and Brun, 2010). Stalk biogenesis and surface attachment must be tightly regulated during the cell cycle, in particular, under environmental conditions (Fiebig et al., 2014), and our work revealed that Lon-mediated proteolysis contributes to this by regulating the stability of StaR and likely other proteins involved in this process, such as the histidine phosphotransferase ShpA and the polysaccharide biosynthesis protein CCNA_02361 that we identified as putative Lon substrates in our proteomics screen (Figure 1F). The gene encoding CCNA_02361 (CC_2278), which was previously shown to contribute to surface attachment (Sprecher et al., 2017), shows a similar cell cycle-dependent pattern in mRNA levels and ribosome occupancy as staR (Lasker et al., 2016; Schrader et al., 2016), with the highest mRNA abundance and translation rate in the swarmer state (Figure 1F). Thus, Lon may also contribute to temporally regulating the abundance of this protein during the cell cycle. 


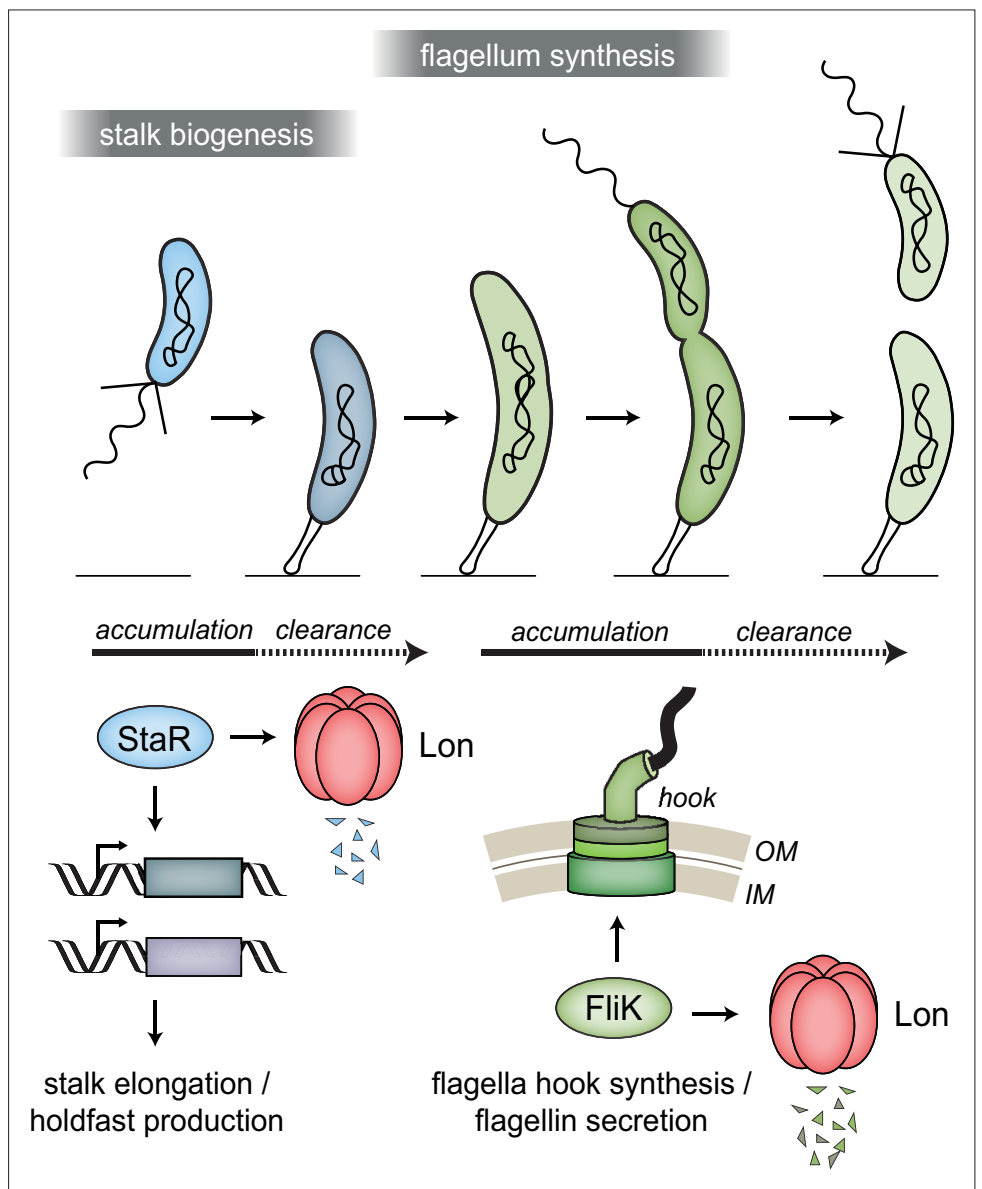

Figure 6. Lon ensures temporal regulation of stalk and flagella biogenesis during the Caulobacter crescentus cell cycle. Lon specifically degrades StaR, a transcriptional regulator of stalk biogenesis and holdfast production, and FliK, a protein involved in regulating flagella hook synthesis. The expression of staR peaks in swarmer cells, while the expression of fliK peaks in late stalked and predivisional cells (Lasker et al., 2016; Laub et al., 2000; Schrader et al., 2016). Our study shows that Lon-dependent proteolysis is required to rapidly eliminate these proteins when their expression levels drop, thus outpacing synthesis. The combination of proteolysis and regulated transcription ensures that StaR abundance is temporally restricted to the swarmer-to-stalked cell transition (shown in blue) and FliK abundance to the late stalked and predivisional cell (shown in green) when their functions are needed for stalk biogenesis or flagella synthesis, respectively.

The online version of this article includes the following figure supplement(s) for figure 6:

Figure supplement 1. FlgE degradation is partially dependent on Lon.

Figure supplement 1-source data 1. Unprocessed Western blot images.

In addition to proteins involved in stalk biogenesis and surface attachment, we identified several proteins required for flagella-mediated motility and chemotaxis as potential Lon substrates (Figure 1F), and investigated FliK in detail. FliK proteins are thought to function as molecular rulers that, while being exported themselves, precisely measure flagellar hook length via the $\mathrm{N}$-terminal domain and mediate via the C-terminal domain a switch from export of hook protein to filament protein, once the hook has reached a certain length (Erhardt et al., 2011; Minamino, 2018; Minamino et alı, 1999; Moriya et al., 2006; Shibata et al., 2007). Although previous work made significant progress in understanding the molecular function of this important protein in enterobacteria, proteolytic control mechanisms contributing to Flik regulation have so far not been described. Moreover, in bacteria outside the gamma-proteobacteria FliK remains poorly studied. In C. crescentus, a fliK gene has previously not been annotated, and our new data revealed that fliK corresponds to a gene that was previously annotated as two separate but overlapping genes. We demonstrate that Lon-mediated proteolysis ensures cell cycle-dependent accumulation of FliK during late S-phase, when the cell prepares for cell 
division by building a new flagellum and chemotaxis apparatus at the swarmer pole (Figure 6). The results that overexpression of FliK results in reduced flagellin levels and impaired motility (Figure 5E and $\mathbf{G}$ ) indicate that this precise regulation of FliK abundance is critical for correct flagella biosynthesis. Based on studies in other bacteria (Muramoto et al., 1998), we consider it possible that an excess of FliK protein causes a premature termination of hook synthesis, leading to shorter hooks, which may cause downstream effects, namely reduced flagellin levels and motility. Our observation that overexpression of FliK-C, a truncated version of FliK lacking the N-terminal domain, which is required for export in other bacteria (Hirano et al., 2005), exhibits an even stronger effect on flagellin levels and motility may be explained by a dominant-negative effect that the truncated version of FliK has over the WT. In this scenario, overexpressed FliK-C would block the normal function of native FliK, possibly by occupying binding sites on interacting proteins, for example, the export apparatus protein FlhB (Kinoshita et al., 2017; Minamino et al., 2009). Our result that deletion of the C-terminal five amino acids of Caulobacter FliK-C abolishes the negative overexpression effects (Figure $\mathbf{5 F}$ and $\mathbf{G}$ ) is consistent with the finding that FliK's export switch-inducing function depends on its C-terminal five amino acids in other bacteria (Kinoshita et al., 2017; Minamino et al., 2006; Williams et al., 1996). Importantly, we also uncovered a critical role of the C-terminal five amino acids of FliK for proteolytic control (Figure 4G), thus, our data indicate a tight coupling between Flik function and degradation.

Interestingly, in addition to the flagella hook length regulator FliK, our data indicate that the flagella hook protein FlgE itself is regulated by Lon in C. crescentus. FlgE was among the proteins that satisfied two criteria in our proteomics approach, and we verified by Western blot analysis that FlgE degradation partly depends on Lon (Figure 6-figure supplement 1). This finding is also consistent with previous work showing that Lon degrades FlgE in E. coli (Arends et al., 2018), and reinforces the previously made notion that precise regulation of the cellular concentrations of FlgE as well as FliK is an important requirement for the correct temporal order of flagella assembly (Inoue et al., 2018).

In several other bacteria, including important human pathogens, Lon has been linked to flagellamediated motility (Ching et al., 2019; Clemmer and Rather, 2008; Fuchs et al., 2001; Rogers et al., 2016). In many of these cases the nature of substrates mediating the observed Lon-dependent effects on motility remains unknown. However, in Bacillus subtilis, it was shown that Lon specifically degrades the master regulator of flagellar biosynthesis SwrA by a mechanism requiring the adaptor SmiA (Mukherjee et al., 2015). In contrast to SwrA, both StaR and FliK are robustly degraded by Lon in vitro (Figures 2D and 4F, Figure 4-figure supplement 2). While these data suggest that no adaptors or other accessory proteins are required to mediate the interaction between these proteins and Lon, it is possible that additional factors exist that modulate the rate of Lon-mediated proteolysis of these proteins in response to specific conditions.

In conclusion, our work provides new insights into the cellular roles of Lon and emphasizes the importance of proteolysis in adjusting the amounts of regulatory proteins involved in critical cellular processes, including cell differentiation and cell cycle progression. Importantly, in addition to StaR and Flik our work identified many other proteins as putative Lon substrates and the precise role of Lon in the regulation of these proteins will be worthwhile to investigate in detail in future studies. Furthermore, our work highlights quantitative proteomics using isobaric mass tags as a powerful approach for the identification of novel protease substrates that could be exploited to identify candidate substrates under diverse growth conditions, in different species or of other proteases.

\section{Materials and methods}

Key resources table
Reagent type

(species) or

resource

Designation

Source or reference

Identifiers

Additional information
Gene (Caulobacter

crescentus) flik-C; motD; CCNA_00945 GeneBank

GeneBank:CCNA_00945

Gene

(C. crescentus) staR; CCNA_02334

GeneBank

GeneBank:CCNA_02334

Continued on next page 
Continued

\section{Reagent type \\ (species) or \\ resource}

Designation

Source or reference

Other

background

(Escherichia coli) DH5 $\alpha$

Strain, strain

background ( $E$.

coli)

BL21-SI/pCodonPlus

Other

Strain, strain

background

(C. crescentus) NA1000 Other

Genetic reagent

(plasmid)

pBX-MCS-4 (plasmid)

Thanbichler et al., 2007

Identifiers

Additional information

Michael Laub, Massachusetts

Institute of Technology;

Chemical competent cells

Claes Andréasson, Stockholm

University;

Electrocompetent cells

Michael Laub, Massachusetts

Institute of Technology;

Electrocompetent cells

Martin Thanbichler, MPI

Marburg

Genetic reagent

(plasmid) pSUMO-YHRC

Holmberg et al., 2014

RRID:Addgene_54336

Goat anti-mouse lgG $(\mathrm{H}+\mathrm{L})$

Antibody Secondary Antibody, HRP

Goat anti-Rabbit lgG $(\mathrm{H}+\mathrm{L})$

Antibody Secondary Antibody, HRP

ANTI-FLAG M2 antibody

Antibody (Mouse monoclonal)

Thermo Fisher Scientific

Cat\# 32430; RRID:AB_1185566

$(1: 5000)$

Antibody

Anti-DnaA (Rabbit polyclonal)

Thermo Fisher Scientific

Cat\# 32460; RRID:AB_1185567

$(1: 5000)$

Cat\# F1804; RRID:AB_262044

$(1: 5000)$

Antibody

Anti-Lon (Rabbit polyclonal) Other

Antibody

Anti-CcrM (Rabbit polyclonal)

Stephens et al., 1996

Gora et al., 2010

(1:5000)

Antibody

Anti-SciP (Rabbit polyclonal)

Davis et al., 2013

Antibody

Anti-TipF (Rabbit polyclonal)

Fiebig et al., 2014

$(1: 10,000)$ kindly provided by

R.T. Sauer

Antibody

Anti-StaR (Rabbit polyclonal)

This paper

$(1: 5000)$

Antibody

Anti-FliK-C (Rabbit polyclonal)

Brun and Shapiro, 1992

Anti-flagellin (Rabbit

Antibody polyclonal)

Peptide,

recombinant

protein

Ulp1-6xHis

Other

$(1: 2000)$

(1:5000) kindly provided by $P$. Viollier

$(1: 500)$

$(1: 500)$

(1:2000) kindly provided by $Y$. Brun

Source vector and purification protocol kindly provided by Claes Andréasson (Stockholm University)

Commercial assay SuperSignal West Femto or kit

Maximum Sensitivity Substrat

Software,

algorithm

Image Lab

Thermo Fisher Scientific

Cat \# 34095

Bio-Rad https://www.bio-rad.

com/en-ca/product/image-lab-

software

RRID:SCR_014210

Version 6.0

Software,

algorithm

GraphPad Prism

https://www.graphpad.com

RRID:SCR_002798

Version 7.0

Software,
algorithm

algorithm Fiji (ImageJ)

Schindelin et al., 2012

https://fiji.sc/

RRID:SCR_002285

4-20\% Mini-PROTEAN TGX

Stain-Free Protein Gels, 15

Other well, $15 \mu$

Bio-Rad

Cat \# 4568096

Other

Trans-Blot Turbo System

Bio-Rad

Cat \# 1704150EDU

Other

LI-COR Odyssey Fc Imaging LI-COR System https://www.licor.com/bio/ odyssey-fc/

(n)




\section{Strains and plasmids}

All bacterial strains, plasmids, and primers used in this study are listed in Supplementary file 1.

\section{Plasmid construction}

\section{Expression plasmids for protein purification}

Plasmids used for protein expression are based on the PSUMO-YHRC backbone and were constructed as follows: the coding sequences of the staR (pMF56-c88) and fliK-C (formerly motD; pMF61) genes were amplified from C. crescentus NA1000 genomic DNA with the primers listed in Supplementary file 1 (see sheets listing primers and vector fragments for sequences and primer combinations, respectively). The backbone vector pSUMO-YHRC was amplified in two parts disrupting the kanamycin resistance gene in order to reduce background (using primer pairs oMJF34/oMJF36 and oMJF37/oMJF38, Supplementary file 1). Following the PCR, the template was digested with Dpnl $(10 \mathrm{U})$ and the remaining PCR fragments were subsequently purified by gel extraction. Fragments were then assembled using Gibson assembly (Gibson et al., 2009). Vectors containing deletions of an annotated gene (FliK-C truncations: pMF66, pMF67-A, and pMF68-A) were derived from vectors harboring the full-length coding sequence in a similar manner using the primer pairs specified in Supplementary file 1.

Replicating plasmids pDJO145 (pBX-MCS-4 containing Ndel-3xFLAG-Kpnl): plasmid pBX-MCS-4 (Thanbichler et al., 2007) was amplified using primers oDJO13 and oDJO41. The sequence encoding the triple FLAG tag (3xFLAG; GAC TAC AAA GAC CAT GAC GGT GAT TAT AAA GAT CAT GAC ATC GAC TAC AAG GAC GAC GAC GAC AAG) was amplified from a plasmid using primers oDJO42 and oDJO43 adding a Kpnl-site followed by a stop codon to the $3^{\prime}$ end of $3 x F L A G$. The two amplified fragments were then joined by Gibson assembly (Gibson et al., 2009).

pDJO151 (pBX-MCS-4 containing $P_{x y l}$-staR-3xFLAG): staR was amplified with primers oDJO44 and oDJO45 using chromosomal C. crescentus NA1000 DNA as template and cloned into Ndel-cut pDJO145 using Gibson assembly.

pDJO157 (pBX-MCS-4 containing $P_{x y l}$-3xFLAG-staR): staR was amplified with primers oDJO46 and oDJO47 using chromosomal C. crescentus NA1000 DNA as template and cloned into Kpnl-cut pDJO145 using Gibson assembly.

pDJO173 (pBX-MCS-4 containing $\left.P_{x y l}-f l g E-3 x F L A G\right)$ : flgE was amplified with primers oDJO65 and oDJO66 using chromosomal C. crescentus NA1000 DNA as template and cloned into Ndel-cut pDJO145 using Gibson assembly.

pDJO200 (pBX-MCS-4 containing P $\left.P_{x y l}-3 x F L A G-f l i K-C\right)$ : fliK-C (CCNA_00945) was amplified with primers oDJO87 and oDJO88 using chromosomal C. crescentus NA1000 DNA as template and cloned into Kpnl-cut pDJO145 using Gibson assembly.

pDJO410 (pBX-MCS-4 containing $P_{x y l}-3 x F L A G-f l i K-C \Delta 5$ ): fliK-C $\Delta 5$ was amplified with primers oDJO87 and oDJO179 using C. crescentus NA1000 DNA as template and cloned into Kpnl-cut pDJO145 using Gibson assembly.

pDJO487 (pBX-MCS-4 containing $P_{x y l}$-3xFLAG-fliK): fliK was amplified with primers oDJO75 and oDJO88 using chromosomal C. crescentus NA1000 DNA as template and cloned into Kpnl-cut pDJO145 using Gibson assembly.

\section{Strain construction}

To generate the $\Delta$ staR $\Delta$ lon strain (KJ1037), the $\Delta$ staR deletion was introduced into the $\Delta$ lon strain (KJ546) by two-step recombination (Skerker et al., 2005) after transformation with plasmid pNTPS138$\triangle$ staR (pAF491; Fiebig et al., 2014). Briefly, transformants were selected on kanamycin plates, single colonies were grown overnight in PYE and plated on PYE containing sucrose. Single sucrose-resistant colonies were subsequently screened for kanamycin sensitivity and the staR knockout was confirmed by colony PCR using primers oDJO40 and oDJO38.

C. crescentus strains carrying replicating plasmids were created by transforming the plasmids into the respective strain backgrounds by electroporation. 


\section{Standard growth conditions}

C. crescentus strains were routinely grown at $30^{\circ} \mathrm{C}$ in PYE medium while shaking at $200 \mathrm{rpm}$ and, if necessary, regularly diluted to assure growth in the exponential phase. If required, the medium was supplemented with xylose ( $0.3 \%$ final), glucose $(0.2 \%$ final), or vanillate $(500 \mu \mathrm{M}$ final). Antibiotics were used at following concentration (liquid/solid media): gentamycin 0.625/5 $\mu \mathrm{g} / \mathrm{ml}$, chloramphenicol $1 / 1 \mu \mathrm{g} / \mathrm{ml}$, and oxytetracycline $1 / 2 \mu \mathrm{g} / \mathrm{ml}$. Experiments were generally performed in the absence of antibiotics when using strains in which the resistance cassette was integrated into the chromosome. For phosphate starvation experiments, log-phase cells grown in M2G (minimal medium with $0.2 \%$ glucose) were washed and transferred to M5G medium lacking phosphate.

E. coli strains for cloning purposes were grown in LB medium at $37^{\circ} \mathrm{C}$, supplemented with antibiotics at following concentrations (liquid/solid media): chloramphenicol 20/40 $\mu \mathrm{g} / \mathrm{ml}$, gentamycin $15 / 20 \mu \mathrm{g} / \mathrm{ml}$, kanamycin $30 / 50 \mu \mathrm{g} / \mathrm{ml}$, and oxytetracyclin $12 / 12 \mu \mathrm{g} / \mathrm{ml}$.

\section{Synchronization of $C$. crescentus cultures}

To synchronize C. crescentus cultures, cells were pelleted by centrifugation at $8000 \mathrm{rpm}$ for $4 \mathrm{~min}$ at $4^{\circ} \mathrm{C}$. The supernatant was aspirated, and tubes were kept on ice. Pellets were resuspended in $1 \mathrm{ml}$ of $1 \times \mathrm{M} 2$ salts on ice. $1 \mathrm{ml}$ of cold Percoll was added and samples were mixed well. The mixture was aliquoted into two Eppendorf tubes and centrifuged at $10,000 \times \mathrm{g}$ for $20 \mathrm{~min}$ at $4^{\circ} \mathrm{C}$. The top layer of the cells was aspirated, and the swarmer cells were moved into a new tube. Swarmer cells were washed twice with $1 \mathrm{ml}$ of cold $1 \times \mathrm{M} 2$ salts and finally resuspended in $20 \mathrm{ml}$ prewarmed PYE medium containing antibiotic if required. Samples were taken immediately after resuspending the cell pellet and subsequently at the indicated time points for immunoblot analysis.

\section{Immunoblot analysis}

For whole-cell extract analysis, $1 \mathrm{ml}$ culture samples were collected after the indicated treatments and time points, and cell pellets were obtained by centrifugation. Cell pellets were resuspended in appropriate amounts of $1 \times$ SDS sample buffer, to ensure normalization of the samples by units $\mathrm{OD}_{600}$ of the cultures. Samples were boiled at $98^{\circ} \mathrm{C}$ for $10 \mathrm{~min}$ and frozen at $-20^{\circ} \mathrm{C}$ until further use. The thawed samples were separated by SDS-PAGE using TGX Stain-free gels (Bio-Rad), and subsequently transferred to nitrocellulose membranes by a semi-dry blotting procedure as per the manufacturer's guidelines. The protein gels and membranes were imaged using a Gel Doc Imager before and after the transfer, respectively, to assess equal loading of total protein as well as the quality of the transfer.

Membranes were blocked in 10\% skim milk powder in TBS-Tween (TBST) and protein levels were detected using the following primary antibodies and dilutions in 3\% skim milk powder in TBST: antiCcrM 1:5000 (Stephens et al., 1996), anti-DnaA 1:5000 (Jonas et al., 2011), anti-Lon 1:10,000 (kindly provided by R.T. Sauer), anti-FLAG M2 antibody 1:5000 (Sigma-Aldrich), anti-SciP 1:2000 (Gora et al., 2010), anti-flagellin 1:2000 (kindly provided by Y. Brun) (Brun and Shapiro, 1992), anti-FliK-C 1:500, anti-StaR 1:500 (Fiebig et al., 2014), and anti-TipF 1:5,000 (kindly provided by P. Viollier) (Davis et al., 2013). Secondary antibodies, 1:5000 dilutions of anti-rabbit or anti-mouse HRP-conjugated antibodies (Thermo Fisher Scientific), and SuperSignal Femto West (Thermo Fisher Scientific) were used to detect primary antibody binding. Immunoblots were scanned using a Chemidoc (Bio-Rad) system or an LI-COR Odyssey Fc system. Relative signal intensities were quantified using the Image Lab software package (Bio-Rad) or ImageJ software.

\section{In vivo degradation assay}

To assess protein degradation in vivo, cells were grown under the appropriate conditions (e.g., for 1-2 hr in the presence of xylose to induce expression of FLAG-tagged proteins), and subsequently protein synthesis was shut off by addition of chloramphenicol $(100 \mu \mathrm{g} / \mathrm{ml})$ or tetracycline $(10 \mu \mathrm{g} / \mathrm{ml})$. Samples were taken at the indicated time points and snap frozen in liquid nitrogen before preparation for analysis by Western blot analysis.

\section{Quantitative proteomics}

Sample preparation was performed as previously described (Schramm et al., 2017). In brief, two independent cultures for each analyzed condition were harvested by centrifugation. Cell pellets were washed using cold $\mathrm{dd}_{2} \mathrm{O}$ and stored at $-80^{\circ} \mathrm{C}$. Protein digestion, TMT10 plex isobaric labeling, and 
the mass spectrometrical analysis were performed by the Clinical Proteomics Mass Spectrometry Facility, Karolinska Institute/Karolinska University Hospital/Science for Life Laboratory.

To identify putative Lon substrates, first, the protein abundances for each condition within one biological replicate were considered to calculate the following ratios (see Figure 1-source data 1): protein levels in $\Delta$ lon at $\mathrm{t}=0 \mathrm{~min} /$ protein levels in WT at $\mathrm{t}=0 \mathrm{~min}(\Delta / \mathrm{on} 0 / \mathrm{WT} 0$ ) to identify proteins with a higher steady-state level in the absence of Lon; protein levels after Lon depletion/protein levels before Lon depletion (Lon depletion (no van)/lon expression (+van)) to identify differences in protein levels after Lon depletion; protein levels before lon overexpression in the presence of glucose/protein levels after $1 \mathrm{hr}$ xylose-induced lon overexpression (gluc/+xyl $1 \mathrm{hr}$ lon overexpression), to identify proteins that are downregulated by lon overexpression; protein levels in WT at $t=0 \mathrm{~min} /$ protein levels in WT at $\mathrm{t}=30 \mathrm{~min}$ (WT 0 /WT 30), to identify proteins that are degraded in WT cells after protein synthesis shut off. Additionally, we calculated the ratios of protein levels in $\Delta /$ on cells at $\mathrm{t}=0 \mathrm{~min} /$ protein levels in $\Delta$ lon cells at $t=30 \mathrm{~min}$ after protein synthesis shut off ( $\Delta$ lon $0 / \Delta$ lon 30 ). Subsequently, the average of the ratios obtained from the two biological replicates was calculated and used for further analysis. Some proteins, as indicated in Figure 1-source data 1, were detected in only one of the replicate data sets. In these cases, only the ratio from the replicate, in which they were detected, was considered.

Next, we selected all proteins with a ratio of ( $\triangle$ lon 0 NWT 0$) \geq 1.1$ for the group 'higher levels in

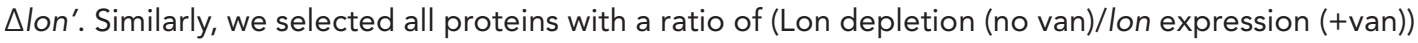
$\geq 1.1$ for the group 'higher levels after Lon depletion'. For the group 'lower levels after lon overexpression', we selected all proteins with a ratio of (gluc/+xyl $1 \mathrm{hr}$ lon overexpression) $\geq 1.05$. For the

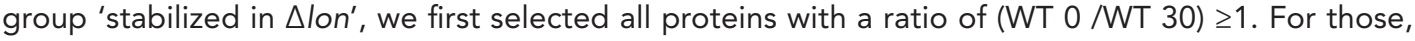
we calculated the ratio of (WT O/WT 30)/( $\Delta$ lon $0 / \Delta$ lon 30 ) and chose the proteins with a ratio $\geq 1.05$. The low threshold ratios were chosen due to a low dynamic range of the data and to ensure that any promising Lon substrate candidates were not missed. To eliminate false positives that may have passed these individual thresholds, an additional filter was applied that at least three of the threshold ratios needed to be met in order to consider a protein a putative Lon substrate. For this, overlaps between the groups of proteins passing the individual thresholds were determined and graphically displayed using jvenn (Bardou et al., 2014; Figure 1D). Functional categories were assigned to the group of putative Lon substrates as well as all detected proteins as previously described (Schramm et al., 2017).

\section{Protein purification}

Protein purification was adapted from Holmberg et al., 2014. In brief, BL21-SI/pCodonPlus cells were transformed using a pSUMO-YHRC derived vector by electroporation. Transformants were selected on $\mathrm{LB}$ agar plates lacking $\mathrm{NaCl}$ (LBON) supplemented with kanamycin and chloramphenicol and pre-cultures inoculated with 20 colonies before being cultivated at $30^{\circ} \mathrm{C}$ overnight. Precultures were diluted 1:100 in $1 \mathrm{~L} 2 x Y T O N$ and grown to $\mathrm{OD}_{600}$ 1.0-1.5. Protein expression was induced with $0.5 \mathrm{mM}$ IPTG and $0.2 \mathrm{M} \mathrm{NaCl}$ either for $4.5 \mathrm{hr}$ at either $30^{\circ} \mathrm{C}$ (StaR and FliK-C) or overnight at $20^{\circ} \mathrm{C}$ (in case of FliK-C truncations). After centrifugation, cell pellets were stored dry at $-80^{\circ} \mathrm{C}$.

Pellets were resuspended in HK500MG (40 mM HEPES-KOH pH 7.5, $500 \mathrm{mM} \mathrm{KCl,} 5 \mathrm{mM} \mathrm{MgCl}$, and $5 \%$ Glycerol) supplemented with $1 \mathrm{mM} \mathrm{PMSF}, 1 \mathrm{mg} / \mathrm{ml}$ Lysozyme, and $3 \mu$ Benzonase/10 $\mathrm{ml}$ suspension and topped up to $20 \mathrm{ml}$. Cells were then lysed by 2-4 passes through an EmulsiFlex-C3 high-pressure homogenizer. Lysate was cleared by centrifugation at $32,500 \times \mathrm{g}$ at $4^{\circ} \mathrm{C}$ for $0.5-1 \mathrm{hr}$. The protein of interest was bound to $1 \mathrm{~g}$ Protino Ni-IDA beads at $4^{\circ} \mathrm{C} / \mathrm{on}$ ice for $30 \mathrm{~min}$. After washing five times with approx. $45 \mathrm{ml}$ HK500MG protein was eluted using HK500MG + $250 \mathrm{mM}$ Imidazole and fractions with protein concentrations $\geq 1 \mathrm{mg} / \mathrm{ml}$ were pooled. For $6 x H i s-S U M O$ tag removal, $4 \mu \mathrm{g} /$ $\mathrm{ml}$ Ulp1-6xHis was added and imidazole was removed in parallel by dialysis against HK500MG. Tag depletion (except for StaR) was achieved by binding to $1 \mathrm{~g}$ Protino Ni-IDA beads as before and flow through containing purified protein was collected. Protein concentration was then checked via SDSPAGE (Bio-Rad 4 -20\% Mini-PROTEAN TGX Stain-Free Protein Gel) and InstantBlue Protein Stain (Expedeon) and quantified using Bio-Rad ImageLab 6.0.1. Protein samples were aliquoted and stored at $-80^{\circ} \mathrm{C}$. 


\section{StaR refolding}

Because StaR was not soluble in neither of the tested buffer conditions and formed precipitates, the protein was refolded (adapted from De Bernardez Clark et alo, 1999; Thomson et alo, 2012). The precipitate was collected (centrifugation at $7197 \times g, 4^{\circ} \mathrm{C}$ ) and solubilized in buffer $\mathrm{S}(50 \mathrm{mM}$ HEPES $\mathrm{pH}$ 8.0, $6 \mathrm{M}$ guanidinium- $\mathrm{HCl}, 1 \mathrm{mM}$ EDTA, and $10 \mathrm{mM} \mathrm{DTT}$ ) and the protein concentration was adjusted to $0.2 \mathrm{mg} / \mathrm{ml}$. The protein solution was then diluted with an equal volume dialysis buffer D1 (50 mM HEPES pH 8.0, 2 M guanidinium- $\mathrm{HCl}$, and $2 \mathrm{mM}$ EDTA) and dialyzed against 125 volumes dialysis buffer D1 followed by dialysis against 125 volumes dialysis buffer D2 (50 mM HEPES pH 8.0, $1 \mathrm{M}$ guanidinium- $\mathrm{HCl}, 2 \mathrm{mM}$ EDTA, $0.4 \mathrm{M}$ Sucrose, $500 \mathrm{mM} \mathrm{KCl}$, and $2 \mathrm{mM} \mathrm{DTT})$. The dialysis buffer was then diluted with one buffer volume of dialysis buffer D3 (50 mM HEPES pH 8.0, 2 mM EDTA, 0.4 M Sucrose, $500 \mathrm{mM} \mathrm{KCl}$, and $2 \mathrm{mM} \mathrm{DTT}$ ) and dialyzed. This was followed by a final dialysis against 125 volumes of dialysis buffer D3 to remove the remaining guanidinium- $\mathrm{HCl}$. Each dialysis step was carried out at $4^{\circ} \mathrm{C}$ for approx. $24 \mathrm{hr}$.

Afterward, insoluble StaR molecules were removed by centrifugation $\left(20,000 \times \mathrm{g}, 4^{\circ} \mathrm{C}, 10 \mathrm{~min}\right)$ and cleared refolded StaR was supplemented with additional $2 \mathrm{mM}$ DTT and concentrated using a centrifugal filter with a MWCO of $3 \mathrm{kDa}(\mathrm{VWR} \# 516-0227 \mathrm{P})$ and stored at $-80^{\circ} \mathrm{C}$. The final concentration was determined by SDS-PAGE using a BSA standard and visualized by InstantBlue Protein Stain (Expedeon).

\section{FliK-C antibody production}

Purified Flik-C was used as antigen to generate rabbit polyclonal antisera (Davids Biotechnologie $\mathrm{GmbH}$ ).

\section{In vitro degradation assays}

In vitro degradation assays were performed as published previously (Jonas et al., 2013). The reaction was carried out in Lon reaction buffer ( $25 \mathrm{mM}$ Tris $\mathrm{pH} 8.0,100 \mathrm{mM} \mathrm{KCl}, 10 \mathrm{mM} \mathrm{MgCl}$, and $1 \mathrm{mM}$ DTT) employing $0.75 \mu \mathrm{M}$ Lon $\left(0.125 \mu \mathrm{M}\right.$ Lon $\left._{6}\right), 4 \mu \mathrm{M}$ substrate (if not stated otherwise), and an ATP regeneration system ( $4 \mathrm{mM}$ ATP, $15 \mathrm{mM}$ creatine phosphate, and $75 \mu \mathrm{g} / \mathrm{ml}$ creatine kinase). The reaction and the ATP regeneration system were prepared separately pre-warmed to $30^{\circ} \mathrm{C}$ (approx. $4 \mathrm{~min}$ ). The reaction was started by adding the ATP regeneration system. Samples were taken at indicated time points and quenched by 1 vol. $2 \times$ SDS sample buffer $(120 \mathrm{mM}$ Tris-Cl pH 6.8, 4\% SDS, 20\% glycerol, and $0.02 \%$ bromophenol blue) and snap frozen in liquid nitrogen. Samples were boiled at $65^{\circ} \mathrm{C}$ for $10 \mathrm{~min}$ and separated by SDS-Page (Bio-Rad 4-20\% Mini-PROTEAN TGX Stain-Free Protein Gel), visualized by InstantBlue Protein Stain (Expedeon) and quantified using Bio-Rad ImageLab 6.0.1. Substrate levels were normalized to Lon and/or creatine kinase levels (in case of '-Lon' samples).

\section{Microscopy}

Cells were fixed by addition of formaldehyde ( $1 \%$ final) to culture samples and stored at $4^{\circ} \mathrm{C}$. For visualization, fixed cells were transferred onto $1 \%$ agarose pads attached to glass slides, covered with a coverslip, and transferred to the microscope. A Ti eclipse inverted research microscope (Nikon) with $100 \times / 1.45$ numerical aperture (NA) objective (Nikon) was used to collect phase-contrast images. The images were processed with Fiji (ImageJ).

\section{Stalk length measurements}

To quantify the stalk length of cells, microscopy images were analyzed using ImageJ. Briefly, the scale was set to one pixel representing an equivalent of $0.0646 \mu \mathrm{m}$. The stalk was then manually selected and length was determined (Figure 3-figure supplement 1). For half-automated analysis (Figure 3E), the software BacStalk was used (Hartmann et al., 2020). The data files containing microscopic images were added to the program, the channel 'phase contrast' was selected, and the scale was set to $1 \mathrm{px} \triangleq 0.0646 \mu \mathrm{m}$. Stalk detection was activated by the setting 'My cells have stalks'. For cell detection, the default settings were used.

\section{Motility assays}

To assess motility, strains were grown in PYE media, supplemented with gentamycin to maintain replicating plasmids when necessary, and cultures were diluted to an $\mathrm{OD}_{600}$ of 0.1 . Subsequently, $1 \mu \mathrm{l}$ of 
each sample was injected about $2 \mathrm{~mm}$ vertically into PYE soft agar plates $(0.35 \%)$, supplemented with gentamycin or gentamycin and xylose when indicated, using a pipette. The plates were incubated at $30^{\circ} \mathrm{C}$ and pictures were taken with the setting 'Blots: Colorimetric' using a ChemiDoc (Bio-Rad).

\section{Acknowledgements}

The authors thank Peter Chien and his lab for sharing aliquots of purified Lon and for their help with Lon purifications, members of the Jonas lab for discussions and specifically Roya Akar for technical assistance, Claes Andréasson and his lab for providing the BL21-SI/pCodonPlus strain and the pSUMO-YHRC vector and for their help with the His-SUMO protein purification procedures, Yves Brun and Patrick Viollier for sharing aliquots of antibodies and Sean Crosson for providing plasmids and the $\triangle$ staR strain. The authors also thank the Clinical proteomics facility at $\mathrm{KI} / \mathrm{KS}$ for support and advice as well as the Protein Expression and Characterization facility at SciLifeLab for sharing their equipment and providing help with the protein purifications. The study was financially supported by grants from the Swedish Research Council (Dnr. 2016-03300 and 2020-03545), the future leaders grant from the Swedish Foundation for Strategic Research (FFL15-0005), and funding from the Strategic Research Area (SFO) program distributed through Stockholm University.

\section{Additional information}

Funding

\begin{tabular}{lll} 
Funder & Grant reference number & Author \\
\hline Vetenskapsrådet & $2016-03300$ & Kristina Jonas \\
\hline Vetenskapsrådet & $2020-03545$ & Kristina Jonas \\
\hline $\begin{array}{l}\text { Swedish Foundation for } \\
\text { Strategic Research }\end{array}$ & FFL15-0005 & Kristina Jonas \\
\hline Stockholm University & SFO funding & Kristina Jonas \\
\hline
\end{tabular}

The funders had no role in study design, data collection and interpretation, or the decision to submit the work for publication.

Author contributions

Deike J Omnus, Matthias J Fink, Conceptualization, Data curation, Formal analysis, Investigation, Methodology, Resources, Validation, Visualization, Writing - original draft, Writing - review and editing; Klaudia Szwedo, Data curation, Formal analysis, Investigation, Writing - review and editing; Kristina Jonas, Conceptualization, Formal analysis, Funding acquisition, Investigation, Project administration, Resources, Supervision, Validation, Visualization, Writing - original draft, Writing - review and editing

Author ORCIDs

Deike J Omnus (iD http://orcid.org/0000-0002-4091-4291

Matthias J Fink (iD http://orcid.org/0000-0002-4620-2009

Kristina Jonas (iD http://orcid.org/0000-0002-1469-4424

Decision letter and Author response

Decision letter https://doi.org/10.7554/73875.sa1

Author response https://doi.org/10.7554/73875.sa2

\section{Additional files}

Supplementary files

- Supplementary file 1. List of bacterial strains, plasmids and primers used in this study.

- Transparent reporting form 
Data availability

All data generated or analysed during this study are included in the manuscript and supporting files.

The following previously published datasets were used:

\begin{tabular}{lllll}
\hline Author(s) & Year & Dataset title & Dataset URL & Database and Identifier \\
\hline Schrader JM, Li G, & 2016 & Ribosome Profiling Reveals & https:/www.ncbi. & NCBI Gene Expression \\
Jonathan WS, Lucy S & & $\begin{array}{l}\text { Translational Control } \\
\text { During the Caulobacter } \\
\text { crescentus cell cycle }\end{array}$ & $\begin{array}{l}\text { nlm.nih.gov/geo/ } \\
\text { query/acc.cgi?acc= }\end{array}$ & Omnibus, GSE68200 \\
& & GSE200 &
\end{tabular}

\section{References}

Arends J, Griego M, Thomanek N, Lindemann C, Kutscher B, Meyer HE, Narberhaus F. 2018. An integrated proteomic approach uncovers novel substrates and functions of the Lon protease in Escherichia coli.

Proteomics 18: e1800080. DOI: https://doi.org/10.1002/pmic.201800080, PMID: 29710379

Bardou P, Mariette J, Escudié F, Djemiel C, Klopp C. 2014. JVENN: An interactive Venn diagram viewer. BMC Bioinformatics 15: 293. DOI: https://doi.org/10.1186/1471-2105-15-293, PMID: 25176396

Biondi EG, Skerker JM, Arif M, Prasol MS, Perchuk BS, Laub MT. 2006. A phosphorelay system controls stalk biogenesis during cell cycle progression in Caulobacter crescentus. Molecular Microbiology 59: 386-401. DOI: https://doi.org/10.1111/j.1365-2958.2005.04970.x, PMID: 16390437

Bota DA, Davies KJ. 2016. Mitochondrial Lon protease in human disease and aging: Including an etiologic classification of Lon-related diseases and disorders. Free Radical Biology \& Medicine 100: 188-198. DOI: https://doi.org/10.1016/j.freeradbiomed.2016.06.031, PMID: 27387767

Brun YV, Shapiro L. 1992. A temporally controlled sigma-factor is required for polar morphogenesis and normal cell division in Caulobacter. Genes \& Development 6: 2395-2408. DOI: https://doi.org/10.1101/gad.6.12a. 2395, PMID: 1459461

Burgos R, Weber M, Martinez S, Lluch-Senar M, Serrano L. 2020. Protein quality control and regulated proteolysis in the genome-reduced organism Mycoplasma pneumoniae. Molecular Systems Biology 16: e9530. DOI: https://doi.org/10.15252/msb.20209530, PMID: 33320415

Ching C, Yang B, Onwubueke C, Lazinski D, Camilli A, Godoy VG. 2019. Lon protease has multifaceted biological functions in Acinetobacter baumannii. Journal of Bacteriology 201: e00536-18. DOI: https://doi.org/10.1128/ JB.00536-18, PMID: 30348832

Clemmer KM, Rather PN. 2008. The Lon protease regulates swarming motility and virulence gene expression in Proteus mirabilis. Journal of Medical Microbiology 57: 931-937. DOI: https://doi.org/10.1099/jmm.0.47778-0, PMID: 18628491

Culp E, Wright GD. 2016. Bacterial proteases, untapped antimicrobial drug targets. The Journal of Antibiotics 70: 366-377. DOI: https://doi.org/10.1038/ja.2016.138

Curtis PD, Brun YV. 2010. Getting in the loop: Regulation of development in Caulobacter crescentus. Microbiology and Molecular Biology Reviews 74: 13-41. DOI: https://doi.org/10.1128/MMBR.00040-09, PMID: 20197497

Davis NJ, Cohen Y, Sanselicio S, Fumeaux C, Ozaki S, Luciano J, Guerrero-Ferreira RC, Wright ER, Jenal U, Viollier PH. 2013. De- and repolarization mechanism of flagellar morphogenesis during a bacterial cell cycle. Genes \& Development 27: 2049-2062. DOI: https://doi.org/10.1101/gad.222679.113, PMID: 24065770

De Bernardez Clark E, Schwarz E, Rudolph R. 1999. Inhibition of aggregation side reactions during in vitro protein folding. Methods in Enzymology 309: 217-236. DOI: https://doi.org/10.1016/s0076-6879(99)09017-5, PMID: 10507027

Eggenhofer E, Rachel R, Haslbeck M, Scharf B. 2006. MotD of Sinorhizobium melilot and related alphaproteobacteria is the flagellar-hook-length regulator and therefore reassigned as FLIK. Journal of Bacteriology 188: 2144-2153. DOI: https://doi.org/10.1128/JB.188.6.2144-2153.2006, PMID: 16513744

Erhardt M, Singer HM, Wee DH, Keener JP, Hughes KT. 2011. An infrequent molecular ruler controls flagellar hook length in Salmonella enterica. The EMBO Journal 30: 2948-2961. DOI: https://doi.org/10.1038/emboj. 2011.185, PMID: 21654632

Fang G, Passalacqua KD, Hocking J, Llopis PM, Gerstein M, Bergman NH, Jacobs-Wagner C. 2013. Transcriptomic and phylogenetic analysis of a bacterial cell cycle reveals strong associations between gene co-expression and evolution. BMC Genomics 14: 450. DOI: https://doi.org/10.1186/1471-2164-14-450, PMID: 23829427

Felletti M, Omnus DJ, Jonas K. 2019. Regulation of the replication initiator DnaA in Caulobacter crescentus. Biochimica et Biophysica Acta. Gene Regulatory Mechanisms 1862: 697-705. DOI: https://doi.org/10.1016/j. bbagrm.2018.01.004, PMID: 29382570

Fiebig A, Herrou J, Fumeaux C, Radhakrishnan SK, Viollier PH, Crosson S. 2014. A cell cycle and nutritional checkpoint controlling bacterial surface adhesion. PLOS Genetics 10: e1004101. DOI: https://doi.org/10.1371/ journal.pgen.1004101, PMID: 24465221 
Fuchs T, Wiget $P$, Osterås M, Jenal U. 2001. Precise amounts of a novel member of a phosphotransferase superfamily are essential for growth and normal morphology in Caulobacter crescentus. Molecular Microbiology 39: 679-692. DOI: https://doi.org/10.1046/j.1365-2958.2001.02238.x, PMID: 11169108

Gibson DG, Young L, Chuang RY, Venter JC, Hutchison CA, Smith HO. 2009. Enzymatic assembly of DNA molecules up to several hundred kilobases. Nature Methods 6: 343-345. DOl: https://doi.org/10.1038/nmeth. 1318, PMID: 19363495

Gora KG, Tsokos CG, Chen YE, Srinivasan BS, Perchuk BS, Laub MT. 2010. A cell-type-specific protein-protein interaction modulates transcriptional activity of a master regulator in Caulobacter crescentus. Molecular Cell 39: 455-467. DOI: https://doi.org/10.1016/j.molcel.2010.06.024, PMID: 20598601

Gora KG, Cantin A, Wohlever M, Joshi KK, Perchuk BS, Chien P, Laub MT. 2013. Regulated proteolysis of a transcription factor complex is critical to cell cycle progression in Caulobacter crescentus. Molecular Microbiology 87: 1277-1289. DOI: https://doi.org/10.1111/mmi.12166, PMID: 23368090

Griffith KL, Shah IM, Wolf RE. 2004. Proteolytic degradation of Escherichia coli transcription activators SoxS and MarA as the mechanism for reversing the induction of the superoxide (SoxRS) and multiple antibiotic resistance (Mar) regulons. Molecular Microbiology 51: 1801-1816. DOI: https://doi.org/10.1046/j.1365-2958.2003.03952. x, PMID: 15009903

Grünenfelder B, Rummel G, Vohradsky J, Röder D, Langen H, Jenal U. 2001. Proteomic analysis of the bacterial cell cycle. PNAS 98: 4681-4686. DOI: https://doi.org/10.1073/pnas.071538098, PMID: 11287652

Gur E, Sauer RT. 2008. Recognition of misfolded proteins by Lon, a AAA(+) protease. Genes \& Development 22 : 2267-2277. DOI: https://doi.org/10.1101/gad.1670908, PMID: 18708584

Gur E. 2013. The Lon AAA+ protease. Sub-Cellular Biochemistry 66: 35-51. DOI: https://doi.org/10.1007/ 978-94-007-5940-4_2, PMID: 23479436

Hartmann R, van Teeseling MCF, Thanbichler M, Drescher K. 2020. Bacstalk: A comprehensive and interactive image analysis software tool for bacterial cell biology. Molecular Microbiology 114: 140-150. DOI: https://doi. org/10.1111/mmi.14501, PMID: 32190923

Hirano T, Shibata S, Ohnishi K, Tani T, Aizawa S-I. 2005. N-terminal signal region of FliK is dispensable for length control of the flagellar hook. Molecular Microbiology 56: 346-360. DOI: https://doi.org/10.1111/j.1365-2958. 2005.04615.x, PMID: 15813729

Holmberg MA, Gowda NKC, Andréasson C. 2014. A versatile bacterial expression vector designed for singlestep cloning of multiple DNA fragments using homologous recombination. Protein Expression and Purification 98: 38-45. DOI: https://doi.org/10.1016/j.pep.2014.03.002, PMID: 24631626

Inoue Y, Morimoto YV, Namba K, Minamino T. 2018. Novel insights into the mechanism of well-ordered assembly of bacterial flagellar proteins in Salmonella. Scientific Reports 8: 1787. DOI: https://doi.org/10.1038/s41598018-20209-3, PMID: 29379125

Ishii Y, Sonezaki S, Iwasaki Y, Miyata Y, Akita K, Kato Y, Amano F. 2000. Regulatory role of c-terminal residues of SulA in its degradation by Lon protease in Escherichia coli. Journal of Biochemistry 127: 837-844. DOI: https:// doi.org/10.1093/oxfordjournals.jbchem.a022677, PMID: 10788793

Jonas K, Chen YE, Laub MT. 2011. Modularity of the bacterial cell cycle enables independent spatial and temporal control of DNA replication. Current Biology 21: 1092-1101. DOI: https://doi.org/10.1016/j.cub.2011. 05.040, PMID: 21683595

Jonas K, Liu J, Chien P, Laub MT. 2013. Proteotoxic stress induces a cell-cycle arrest by stimulating Lon to degrade the replication initiator DnaA. Cell 154: 623-636. DOI: https://doi.org/10.1016/j.cell.2013.06.034, PMID: 23911325

Joshi KK, Chien P. 2016. Regulated proteolysis in bacteria: Caulobacter. Annual Review of Genetics 50: $423-445$. DOI: https://doi.org/10.1146/annurev-genet-120215-035235, PMID: 27893963

Joshi A, Mahmoud SA, Kim SK, Ogdahl JL, Lee VT, Chien P, Yildiz FH. 2020. C-di-GMP inhibits LonA-dependent proteolysis of TfoY in Vibrio cholerae. PLOS Genetics 16: e1008897. DOI: https://doi.org/10.1371/journal. pgen.1008897, PMID: 32589664

Kinoshita M, Aizawa S-I, Inoue Y, Namba K, Minamino T. 2017. The role of intrinsically disordered c-terminal region of flik in substrate specificity switching of the bacterial flagellar type III export apparatus. Molecular Microbiology 105: 572-588. DOI: https://doi.org/10.1111/mmi.13718, PMID: 28557186

Langklotz S, Narberhaus F. 2011. The Escherichia coli replication inhibitor CspD is subject to growth-regulated degradation by the Lon protease. Molecular Microbiology 80: 1313-1325. DOI: https://doi.org/10.1111/j. 1365-2958.2011.07646.x, PMID: 21435040

Lasker K, Schrader JM, Men Y, Marshik T, Dill DL, McAdams HH, Shapiro L. 2016. Caulobrowser: A systems biology resource for Caulobacter crescentus. Nucleic Acids Research 44: D640-D645. DOI: https://doi.org/10. 1093/nar/gkv1050, PMID: 26476443

Laub MT, McAdams HH, Feldblyum T, Fraser CM, Shapiro L. 2000. Global analysis of the genetic network controlling a bacterial cell cycle. Science 290: 2144-2148. DOI: https://doi.org/10.1126/science.290.5499. 2144, PMID: 11118148

Leslie DJ, Heinen C, Schramm FD, Thüring M, Aakre CD, Murray SM, Laub MT, Jonas K. 2015. Nutritional control of DNA replication initiation through the proteolysis and regulated translation of DnaA. PLOS Genetics 11: e1005342. DOI: https://doi.org/10.1371/journal.pgen.1005342, PMID: 26134530

Liu J, Francis L, Jonas K, Laub MT, Chien P. 2016. ClpAP is an auxiliary protease for DnaA degradation in Caulobacter crescentus. Molecular Microbiology 102: 1075-1085. DOI: https://doi.org/10.1111/mmi.13537, PMID: 27667502 
McGrath PT, Lee H, Zhang L, Iniesta AA, Hottes AK, Tan MH, Hillson NJ, Hu P, Shapiro L, McAdams HH. 2007. High-throughput identification of transcription start sites, conserved promoter motifs and predicted regulons. Nature Biotechnology 25: 584-592. DOI: https://doi.org/10.1038/nbt1294, PMID: 17401361

Minamino T, González-Pedrajo B, Yamaguchi K, Aizawa SI, Macnab RM. 1999. FliK, the protein responsible for flagellar hook length control in salmonella, is exported during hook assembly. Molecular Microbiology 34: 295-304. DOI: https://doi.org/10.1046/j.1365-2958.1999.01597.x, PMID: 10564473

Minamino T, Ferris HU, Moriya N, Kihara M, Namba K. 2006. Two parts of the T3S4 domain of the hook-length control protein FliK are essential for the substrate specificity switching of the flagellar type III export apparatus. Journal of Molecular Biology 362: 1148-1158. DOI: https://doi.org/10.1016/j.jmb.2006.08.004, PMID: 16949608

Minamino T, Moriya N, Hirano T, Hughes KT, Namba K. 2009. Interaction of FliK with the bacterial flagellar hook is required for efficient export specificity switching. Molecular Microbiology 74: 239-251. DOI: https://doi.org/ 10.1111/j.1365-2958.2009.06871.x, PMID: 19732341

Minamino T. 2018. Hierarchical protein export mechanism of the bacterial flagellar type III protein export apparatus. FEMS Microbiology Letters 365: 12. DOI: https://doi.org/10.1093/femsle/fny117, PMID: 29850796 Mizusawa S, Gottesman S. 1983. Protein degradation in Escherichia coli: the lon gene controls the stability of sulA protein. PNAS 80: 358-362. DOI: https://doi.org/10.1073/pnas.80.2.358, PMID: 6300834

Moriya N, Minamino T, Hughes KT, Macnab RM, Namba K. 2006. The type III flagellar export specificity switch is dependent on FliK ruler and a molecular clock. Journal of Molecular Biology 359: 466-477. DOI: https://doi. org/10.1016/j.jmb.2006.03.025, PMID: 16630628

Mukherjee S, Bree AC, Liu J, Patrick JE, Chien P, Kearns DB. 2015. Adaptor-mediated Lon proteolysis restricts Bacillus subtilis hyperflagellation. PNAS 112: 250-255. DOI: https://doi.org/10.1073/pnas.1417419112, PMID: 25538299

Muramoto K, Makishima S, Aizawa SI, Macnab RM. 1998. Effect of cellular level of FliK on flagellar hook and filament assembly in Salmonella typhimurium. Journal of Molecular Biology 277: 871-882. DOI: https://doi.org/ 10.1006/jmbi.1998.1659, PMID: 9545378

Muthuramalingam M, White JC, Bourne CR. 2016. Toxin-antitoxin modules are pliable switches activated by multiple protease pathways. Toxins 8: E214. DOI: https://doi.org/10.3390/toxins8070214, PMID: 27409636

Phillips TA, VanBogelen RA, Neidhardt FC. 1984. Lon gene product of Escherichia coli is a heat-shock protein. Journal of Bacteriology 159: 283-287. DOI: https://doi.org/10.1128/JB.159.1.283-287.1984, PMID: 6330035

Piovesan D, Necci M, Escobedo N, Monzon AM, Hatos A, Mičetić I, Quaglia F, Paladin L, Ramasamy P, Dosztányi Z, Vranken WF, Davey NE, Parisi G, Fuxreiter M, Tosatto SCE. 2021. MobiDB: Intrinsically disordered proteins in 2021. Nucleic Acids Research 49: D361-D367. DOI: https://doi.org/10.1093/nar/gkaa1058, PMID: 33237329

Puri N, Karzai AW. 2017. HspQ Functions as a Unique Specificity-Enhancing Factor for the AAA+ Lon Protease. Molecular Cell 66: 672-683. DOI: https://doi.org/10.1016/j.molcel.2017.05.016, PMID: 28575662

Rogers A, Townsley L, Gallego-Hernandez AL, Beyhan S, Kwuan L, Yildiz FH. 2016. The LonA Protease Regulates Biofilm Formation, Motility, Virulence, and the Type VI Secretion System in Vibrio cholerae. Journal of Bacteriology 198: 973-985. DOI: https://doi.org/10.1128/JB.00741-15, PMID: 26755629

Sauer RT, Baker TA. 2011. AAA+ proteases: ATP-fueled machines of protein destruction. Annual Review of Biochemistry 80: 587-612. DOI: https://doi.org/10.1146/annurev-biochem-060408-172623, PMID: 21469952

Schindelin J, Arganda-Carreras I, Frise E, Kaynig V, Longair M, Pietzsch T, Preibisch S, Rueden C, Saalfeld S, Schmid B, Tinevez JY, White DJ, Hartenstein V, Eliceiri K, Tomancak P, Cardona A. 2012. Fiji: an open-source platform for biological-image analysis. Nature Methods 9: 676-682. DOI: https://doi.org/10.1038/nmeth.2019, PMID: 22743772

Schmidt JM, Stanier RY. 1966. The development of cellular stalks in bacteria. The Journal of Cell Biology 28: 423-436. DOI: https://doi.org/10.1083/jcb.28.3.423, PMID: 5960805

Schrader JM, Zhou B, Li G-W, Lasker K, Childers WS, Williams B, Long T, Crosson S, McAdams HH, Weissman JS, Shapiro L. 2014. The coding and noncoding architecture of the Caulobacter crescentus genome. PLOS Genetics 10: e1004463. DOI: https://doi.org/10.1371/journal.pgen.1004463, PMID: 25078267

Schrader JM, Li GW, Childers WS, Perez AM, Weissman JS, Shapiro L, McAdams HH. 2016. Dynamic translation regulation in Caulobacter cell cycle control. PNAS 113: E6859-E6867. DOI: https://doi.org/10.1073/pnas. 1614795113, PMID: 27791168

Schramm FD, Heinrich K, Thüring M, Bernhardt J, Jonas K. 2017. An essential regulatory function of the DnaK chaperone dictates the decision between proliferation and maintenance in Caulobacter crescentus. PLOS Genetics 13: e1007148. DOI: https://doi.org/10.1371/journal.pgen.1007148, PMID: 29281627

Schroeder K, Jonas K. 2021. The protein quality control network in Caulobacter crescentus. Frontiers in Molecular Biosciences 8: 682967. DOI: https://doi.org/10.3389/fmolb.2021.682967, PMID: 33996917

Shibata S, Takahashi N, Chevance FF, Karlinsey JE, Hughes KT, Aizawa S. 2007. FliK regulates flagellar hook length as an internal ruler. Molecular Microbiology 64: 1404-1415. DOI: https://doi.org/10.1111/j.1365-2958. 2007.05750.x, PMID: 17542929

Skerker JM, Prasol MS, Perchuk BS, Biondi EG, Laub MT. 2005. Two-component signal transduction pathways regulating growth and cell cycle progression in a bacterium: a system-level analysis. PLOS Biology 3: e334. DOI: https://doi.org/10.1371/journal.pbio.0030334, PMID: 16176121

Sprecher KS, Hug I, Nesper J, Potthoff E, Mahi MA, Sangermani M, Jenal U. 2017. Cohesive Properties of the Caulobacter crescentus Holdfast Adhesin Are Regulated by a Novel c-di-GMP Effector Protein. mBio 8: e00294-17. DOI: https://doi.org/10.1128/mBio.00294-17, PMID: 28325767 
Stein BJ, Fiebig A, Crosson S. 2020. Feedback Control of a Two-Component Signaling System by an Fe-SBinding Receiver Domain. mBio 11: e03383-19. DOI: https://doi.org/10.1128/mBio.03383-19, PMID: 32184258 Stephens C, Reisenauer A, Wright R, Shapiro L. 1996. A cell cycle-regulated bacterial DNA methyltransferase is essential for viability. PNAS 93: 1210-1214. DOI: https://doi.org/10.1073/pnas.93.3.1210, PMID: 8577742

Thanbichler M, Iniesta AA, Shapiro L. 2007. A comprehensive set of plasmids for vanillate- and xylose-inducible gene expression in Caulobacter crescentus. Nucleic Acids Research 35: e137. DOI: https://doi.org/10.1093/ nar/gkm818, PMID: 17959646

Thomson CA, Olson M, Jackson LM, Schrader JW. 2012. A simplified method for the efficient refolding and purification of recombinant human GM-CSF. PLOS ONE 7: e49891. DOI: https://doi.org/10.1371/journal.pone. 0049891, PMID: 23166789

Tomoyasu T, Mogk A, Langen H, Goloubinoff P, Bukau B. 2001. Genetic dissection of the roles of chaperones and proteases in protein folding and degradation in the Escherichia coli cytosol. Molecular Microbiology 40: 397-413. DOI: https://doi.org/10.1046/j.1365-2958.2001.02383.x, PMID: 11309122

Tsilibaris V, Maenhaut-Michel G, Van Melderen L. 2006. Biological roles of the Lon ATP-dependent protease. Research in Microbiology 157: 701-713. DOI: https://doi.org/10.1016/j.resmic.2006.05.004, PMID: 16854568

Wargachuk R, Marczynski GT. 2015. The Caulobacter crescentus homolog of DnaA (HdaA) also regulates the proteolysis of the replication initiator protein DNAA. Journal of Bacteriology 197: 3521-3532. DOI: https://doi. org/10.1128/JB.00460-15, PMID: 26324449

Waters RC, O'Toole PW, Ryan KA. 2007. The FliK protein and flagellar hook-length control. Protein Science 16: 769-780. DOI: https://doi.org/10.1110/ps.072785407, PMID: 17456739

Williams AW, Yamaguchi S, Togashi F, Aizawa SI, Kawagishi I, Macnab RM. 1996. Mutations in FliK and FlhB affecting flagellar hook and filament assembly in Salmonella typhimurium. Journal of Bacteriology 178: 2960-2970. DOI: https://doi.org/10.1128/jb.178.10.2960-2970.1996, PMID: 8631688

Wright R, Stephens C, Zweiger G, Shapiro L, Alley MR. 1996. Caulobacter Lon protease has a critical role in cell-cycle control of DNA methylation. Genes \& Development 10: 1532-1542. DOI: https://doi.org/10.1101/ gad.10.12.1532, PMID: 8666236

Yang Q, Du Y, Haynes CM, Chien P. 2018. A Legacy Role for DNA Binding of Lon Protects against Genotoxic Stress. bioRxiv. DOI: https://doi.org/10.1101/317677

Zhou X, Wang J, Herrmann J, Moerner WE, Shapiro L. 2019. Asymmetric division yields progeny cells with distinct modes of regulating cell cycle-dependent chromosome methylation. PNAS 116: 15661-15670. DOI: https://doi.org/10.1073/pnas.1906119116, PMID: 31315982

Zhou X, Eckart MR, Shapiro L. 2021. A bacterial toxin perturbs intracellular amino acid balance to induce persistence. mBio 12: e03020-20. DOI: https://doi.org/10.1128/mBio.03020-20, PMID: 33622732 